\title{
Drivers of the Primate Thalamus
}

\author{
Zita Rovó, ${ }^{1}$ István Ulbert, ${ }^{2,3}$ and László Acsády ${ }^{1}$ \\ ${ }^{1}$ Laboratory of Thalamus Research, Institute of Experimental Medicine and ${ }^{2}$ Institute of Cognitive Neuroscience and Psychology, Research Centre for \\ Natural Sciences, Hungarian Academy of Sciences, 1083, Budapest, Hungary, and 3Péter Pázmány Catholic University, Faculty of Information Technology, \\ 1083, Budapest, Hungary
}

The activity of thalamocortical neurons is primarily determined by giant excitatory terminals, called drivers. These afferents may arise from neocortex or from subcortical centers; however, their exact distribution, segregation, or putative absence in given thalamic nuclei are unknown. To unravel the nucleus-specific composition of drivers, we mapped the entire macaque thalamus using vesicular glutamate transporters 1 and 2 to label cortical and subcortical afferents, respectively. Large thalamic territories were innervated exclusively by either giant vGLUT2- or vGLUT1-positive boutons. Codistribution of drivers with different origin was not abundant. In several thalamic regions, no giant terminals of any type could be detected at light microscopic level. Electron microscopic observation of these territories revealed either the complete absence of large multisynaptic excitatory terminals (basal ganglia-recipient nuclei) or the presence of both vGLUT1- and vGLUT2-positive terminals, which were significantly smaller than their giant counterparts (intralaminar nuclei, medial pulvinar). In the basal ganglia-recipient thalamus, giant inhibitory terminals replaced the excitatory driver inputs. The pulvinar and the mediodorsal nucleus displayed subnuclear heterogeneity in their driver assemblies. These results show that distinct thalamic territories can be under pure subcortical or cortical control; however, there is significant variability in the composition of major excitatory inputs in several thalamic regions. Because thalamic information transfer depends on the origin and complexity of the excitatory inputs, this suggests that the computations performed by individual thalamic regions display considerable variability. Finally, the map of driver distribution may help to resolve the morphological basis of human diseases involving different parts of the thalamus.

\section{Introduction}

Thalamocortical (TC) information transfer is essential for normal cortical functions: thus, understanding the principles of thalamic relay will help to clarify the basic principles of cortical operation. The "driver theory" is an influential conceptual framework that describes the morphological and functional bases of TC relay (Sherman and Guillery, 1998, 2006). According to the original concept, thalamic activity is primarily determined by specialized giant excitatory terminals (i.e., "drivers") that form multiple release sites on the proximal dendrites of TC cells and secure faithful information transfer (Sherman and Guillery, 1996, 1998, 2006). The origin of drivers was used to divide the thalamus into two regions. "First-order" thalamic relays receive drivers from subcortical centers (retina, spinal cord, cerebellum) and thus transmit visual, somatosensory, motor, etc., information (Jones, 2007), whereas "higher-order" relays are contacted by cortical layer 5 pyramidal cells (Sherman and Guillery, 1996, 2006; Rouiller and Welker, 2000) and can participate in cortico-

\footnotetext{
Received June 13, 2012; revised Sept. 6, 2012; accepted 0ct. 19, 2012.

Author contributions: L.A. designed research; Z.R. and I.U. performed research; Z.R. and L.A. analyzed data; Z.R. and L.A. wrote the paper.

This work was supported by the Wellcome Trust (a Wellcome Project Grant to L.A.), the Hungarian Scientific Research Fund (Grants OTKA T 75676 and OTKA 81357), and National Office for Research and Technology-ANR TéT Funds (Multisca and Neurogen). We thank Krisztina Faddi and Gyöző Goda for their excellent technical assistance and H. Bokor for critical reading of the manuscript.

This article is freely available online through the J Neurosci Open Choice option.

Correspondence should be addressed to László Acsády, Institute of Experimental Medicine, Hungarian Academy of Sciences, P.0. Box 67, 1450 Budapest, Hungary. E-mail: acsady@koki.hu.

DOI:10.1523/JNEUROSCI.2815-12.2012

Copyright $\odot 2012$ the authors $\quad 0270-6474 / 12 / 3217894-15 \$ 15.00 / 0$
}

cortical information transfer via the thalamus (Theyel et al., 2010). According to the core concept, the synaptic properties of cortical and subcortical drivers are similar (Reichova and Sherman, 2004; Groh et al., 2008; Budisantoso et al., 2012), but the content of information to be transferred depends on the origin of the drivers.

The exact distribution of cortical versus subcortical drivers and their potential heterogeneity in the thalamus is unknown. It is not clear whether cortical or subcortical drivers segregate spatially or whether drivers with different origin can intermingle. It has also not been examined whether every thalamic region always receives some type of large excitatory driver terminal or not. Finally, it is an open question whether large excitatory terminals are homogeneous or display nucleus-specific variability.

Recently, the thalamus has become a major target for deepbrain intervention in several major neurological diseases, including essential and Parkinsonian tremors (Limousin et al., 1999), epilepsy (Fisher et al., 2010), chronic pain (Hamani et al., 2006), and the treatment of major disturbances of consciousness (Schiff et al., 2007; Yamamoto et al., 2010). In the majority of these cases, little is known about the connectivity of thalamic target sites, which makes rational intervention difficult. Thus, to facilitate a comparison with the human brain, in this study, we mapped the drivers of the macaque thalamus.

Although tract-tracing methods have provided an important foundation for our understanding of TC networks, they are unable to resolve all afferents at the same time. Thus, we used two well-established neurochemical markers, the vesicular glutamate transporters 1 and 2 (vGLUT1 and vGLUT2), to label cortical and subcortical excitatory inputs in the thalamus, respectively 
(Fremeau et al., 2001; Herzog et al., 2001; Land et al., 2004; Lavallée et al., 2005; Graziano et al., 2008; Masterson et al., 2009). The applicability of these markers was verified by tracing and colocalization methods. Our data show a surprising nucleusspecific heterogeneity of thalamic driver inputs, including the convergence of drivers, the lack of morphologically identifiable drivers, and significant heterogeneity in the morphological complexity of driver terminals.

\section{Materials and Methods}

Surgery. Material from a total of seven adult female macaque monkeys (Macaca mulatta) were used in this study. All experimental procedures were performed according to the ethical guidelines of the Institute of Experimental Medicine and Institute of Physiology of the Hungarian Academy of Sciences and approved by the Ethical Committee (Approval 22.1/77/001/2010).

Three animals received unilateral injections of biotinylated dextran amine [BDA; 10,000 molecular weight (Invitrogen), $10 \%$ in $0.1 \mathrm{M}$ phosphate buffer (PB), pH 7.4]. Aseptic techniques were used during the surgical procedures. Anesthesia was induced by intramuscular injection of ketamine hydrochloride $(10 \mathrm{mg} / \mathrm{kg})$ and xylazine $(1 \mathrm{mg} / \mathrm{kg})$ and then maintained by intravenous administration of ketamine/xylazine. Body temperature was kept at $37-38^{\circ} \mathrm{C}$ with a heating pad. Heart rate and body temperature were continuously monitored throughout the procedure and used to adjust anesthetic depth. The head was held by hollow ear bars affixed to a stereotaxic frame (David Kopf Instruments). A midline incision was made exposing the skull, followed by retraction of the skin. A craniotomy was performed exposing the central sulcus and the superior precentral dimple. After retraction of the dura mater, warm silicone was applied to the brain to prevent desiccation of the cortex. Photographs of the exposed cortical surface were taken for recording the locations of electrode penetrations. BDA was injected via glass micropipettes (tip diameter, $50-70 \mu \mathrm{m}$ ) with positive current pulses ( 2 s duty cycles, $5 \mu \mathrm{A}$ for $20 \mathrm{~min}$ ). After the surgery, the animals were treated with antibiotics and analgesics (cobactan, $2.5 \%, 0.3 \mathrm{ml} / \mathrm{d}$ ).

Two of the injections targeted the primary and supplementary motor areas. In the third animal, the ventral part of the internal capsule was injected to label large populations of layer 5 axons without significant layer 6 labeling (Veinante et al., 2000). After a survival period of 14-20 d, the animals were killed.

Perfusion and preparation of tissue sections. The animals were perfused under deep ketamine/xylazine anesthesia through the heart, first with $500 \mathrm{ml}$ of physiological saline ( $5 \mathrm{~min}$ ), followed by $500 \mathrm{ml}$ of fixative containing $2 \%$ paraformaldehyde (TAAB Laboratories Equipment) and $0.5 \%$ glutaraldehyde (TAAB Laboratories Equipment) in acetate buffer, pH 6.0 (5 min), and finally with $1500 \mathrm{ml}$ of fixative containing 2\% paraformaldehyde and $0.5 \%$ glutaraldehyde in borate buffer, $\mathrm{pH} 8.5$ (60 min) (Berod et al., 1981).

After perfusion, coronal serial sections (50 $\mu \mathrm{m}$ thick) were cut with a vibratome, washed, cryoprotected in $30 \%$ sucrose in $0.1 \mathrm{M} \mathrm{PB}$ overnight, and freeze-thawed in an aluminum boat over liquid nitrogen. After extensive washes and treatment with $1 \%$ borohydride, the sections were processed for immunostaining.

Immunocytochemical procedures. To demonstrate the specificity of the immunostainings, two primary antibodies raised against different epitopes of the vGLUT peptides were used for both vGLUT1 and vGLUT2 immunostainings. These primary antibodies were guinea pig anti-vGLUT1 (1:5000-1:10,000; Millipore Bioscience Research Reagents), rabbit anti-vGLUT1 (1:10,000; Synaptic Systems), mouse antivGLUT2 (1:3000-1:4000; Millipore Bioscience Research Reagents), and rabbit anti-vGLUT2 (1:3000-1:4000; Synaptic Systems). The immunostainings with different antibodies raised against the same vGLUT antigen gave identical results. To delineate intralaminar nuclei, mouse monoclonal anti-calbindin (1:2000; Swant) and rabbit anti-calbindin (1:2000; kind gift from K. G. Baimbridge, University of British Columbia, Vancouver, BC, Canada) were used.

Single immunostainings: light microscopic studies. After incubation with the primary antibodies (see above; overnight, room temperature), the sections were treated with the respective biotinylated secondary antibodies ( $2 \mathrm{~h}$ at 1:300; Vector Laboratories) and then with avidin-biotin horseradish peroxidase complex (ABC; $2 \mathrm{~h}$ at 1:300; Vector Laboratories). All the washes and dilutions of antisera were done in $0.05 \mathrm{M}$ Tris-buffered saline (TBS), pH 7.4. The immunoperoxidase reaction was developed with nickel-intensified 3.3' -diaminobenzidine (DAB-Ni) as a chromogen (black reaction product). For nickel intensification, the following solution was used: $16 \mathrm{ml}$ of $0.1 \mathrm{M} \mathrm{PB}, \mathrm{pH} 7.4,1 \mathrm{ml}$ of $0.5 \%$ DAB solution, $6.6 \mathrm{mg}$ of $\mathrm{NH}_{4} \mathrm{Cl}$, and $0.8 \mathrm{ml}$ of $0.05 \mathrm{M} \mathrm{NiNH}_{4} \mathrm{SO}_{4}$. All sections were postfixed in $1 \% \mathrm{OsO}_{4}$ containing $7 \%$ glucose, followed by dehydration in a series of ethanol at increasing concentrations and acetonitrile and then embedding in Durcupan (ACM; Fluka). BDA was visualized by ABC and DAB-Ni.

Electron microscopic observations. The sections were developed with the silver-intensified gold method. Briefly, after incubation with the primary antibodies, sections were treated with the respective $1 \mathrm{~nm}$ goldconjugated secondary antibodies (1:50; Aurion) diluted in TBS containing $0.8 \%$ bovine serum albumin, $0.05 \%$ sodium-azide, and $0.1 \%$ cold-water fish skin gelatin (Aurion) overnight. After washes in TBS, the sections were treated with $1 \%$ glutaraldehyde diluted in TBS, washed again in TBS, treated with $10 \%$ enhancement conditioning solution (Aurion), and developed with the Aurion R-GENT LM intensification kit. After intensification, all sections were postfixed in ice-cold $\mathrm{OsO}_{4}$ solution $(0.5 \%$ in $\mathrm{PB})$, followed by dehydration and embedding as above. During dehydration, the $70 \%$ ethanol step contained $1 \%$ uranyl acetate (40 min).

Double immunostainings. For double labeling of the two vGLUTs, two combinations were used. For examinations at the light microscopic level, rabbit anti-vGLUT2 (1:3000) and guinea pig anti-vGLUT1 $(1: 10,000)$ were mixed. Next, the sections were treated with anti-rabbit ImmPRESS reagent (1:2 in TBS; Vector Laboratories) and developed with DAB-Ni (black reaction product). This was followed by biotinylated goat antiguinea pig and $\mathrm{ABC}$. The second immunoreaction was visualized by $\mathrm{DAB}$ alone (brown reaction product). The sections were dehydrated as above, and $7 \%$ sucrose was added to the $\mathrm{OsO}_{4}$ solution to preserve color differences.

For electron microscopic analysis, a mixture of rabbit anti-vGLUT1 (1:5000) and mouse anti-vGLUT2 (1:3000) was used. First, vGLUT1 was visualized using gold-conjugated goat anti-rabbit (1:50; Aurion) and silver intensification (see above), followed by vGLUT2 visualization by $\mathrm{ABC}$ and $\mathrm{DAB}$. The sections were prepared for electron microscopic analysis as described above.

For correlated light and electron microscopy of the tracer and vGLUT2, BDA was visualized by $A B C$, and DAB-Ni and vGLUT2 were visualized by anti-rabbit vGLUT2, ImmPRESS, and DAB.

Double fluorescent immunostainings. For simultaneous detection of the tracer and the two vGLUTs, the sections were incubated with rabbit anti-vGLUT1 (1:5000, in 0.5\% Triton X-100, TBS) and mouse antivGLUT2 (1:3000), followed by a treatment with Cy3-conjugated donkey anti-rabbit (1:500; Jackson ImmunoResearch), Cy5 donkey anti-mouse (1:500; Jackson ImmunoResearch), and streptavidin-conjugated Alexa Fluor 488 (1:2000; Invitrogen), or Cy5 donkey anti-rabbit Alexa Fluor 594 goat anti-mouse, and streptavidin-conjugated Alexa Fluor 488. To decrease lipofuscin autofluorescence, the sections were treated for $1 \mathrm{~h}$ with a $\mathrm{CuSO}_{4}$-containing solution according to Schnell et al. (1999). Finally, sections were mounted, coverslipped with Vectashield (Vector Laboratories), and examined with a confocal microscope.

Postembedding immunostaining: semithin sections. Colocalization of vGLUT1 and vGLUT2 was also performed on semithin sections. Briefly, sections were embedded into Durcupan without $\mathrm{OSO}_{4}$ pretreatment. After reembedding, $0.5-\mu \mathrm{m}$-thick sections were cut, mounted on slides, and etched using saturated sodium ethanolate. The immunoreactions were performed in Petri dishes using drops of primary and fluorescent secondary antibodies as described above.

Postembedding immunostaining: GABA immunogold for electron microscopy. After preembedding immunostaining for vGLUT1 and vGLUT2, blocks containing various thalamic nuclei were reembedded. Ultrathin sections were cut with an ultramicrotome and mounted on nickel grids. Postembedding GABA immunostaining was performed on 
nickel grids according to a previously described protocol (Somogyi and Hodgson, 1985). The rabbit anti-GABA antibody (kindly donated by Prof. Péter Somogyi, Medical Research Council, Oxford, UK) was raised against GABA bound to bovine serum albumin. The antibody has been extensively characterized previously (Somogyi et al., 1985). Incubations were performed on droplets of solutions in humid Petri dishes, as follows: $0.5 \%$ periodic acid for $2-5 \mathrm{~min}$; wash in distilled water; three times for $2 \mathrm{~min}$ in TBS; $30 \mathrm{~min}$ in 1\% ovalbumin dissolved in TBS; three times for $10 \mathrm{~min}$ in TBS, $1-2 \mathrm{~h}$ in a rabbit anti-GABA antiserum (1:6000-1: 8000 in TBS); two times for $10 \mathrm{~min}$ in TBS; $10 \mathrm{~min}$ in Tris buffer containing $1 \%$ bovine serum albumin, $0.05 \%$ Tween $20 ; 2 \mathrm{~h}$ in colloidal gold-conjugated goat anti-rabbit IgG [12 nm (Jackson ImmunoResearch), 1:20, or $15 \mathrm{~nm}$ (GE Healthcare), 1:20 in the same solution as before); two times for $5 \mathrm{~min}$ wash in distilled water; 20 min saturated uranyl acetate; wash in distilled water; staining with lead citrate; wash in distilled water. Profiles were considered GABA positive if they contained five times higher density of gold particles than background labeling as measured above for the cell bodies of relay cells. The etching procedure may remove some silver precipitate, so only every second grid was reacted for postembedding immunostaining.

Image acquisition and processing. Digital light micrographs were taken with an AxioCam HRC (Carl Zeiss) connected to AxioImager M1 microscope (Carl Zeiss). Digital montages of serial photos taken through the thickness of a section were processed with the "extended depth of field function" of Image-Pro Express 6.0 (Media Cybernetics). Confocal images were acquired with a confocal laser scanning microscope (A1R; Nikon) in sequential mode and a CFI Plan Apochromat VC lens $60 \times$ (numerical aperture 1.40) with a pinhole set at 1 Airy unit and a pixel size of $90 \mathrm{~nm}$. Electron micrographs were taken by a side-mounted Veleta CCD camera (Olympus Soft Imaging Solutions) connected to an Hitachi $\mathrm{H}-7100$ electron microscope. In the digital images, brightness and contrast were adjusted when necessary, applied to whole images only, using Adobe Photoshop CS2 (Adobe Systems).

Data analyses. The borders of the thalamus were drawn by camera lucida at low magnification. The exact ventral borders with zona incerta and the caudomedial border with the brainstem were established by the characteristic dense vGLUT1 immunostaining of the thalamus. The distribution of large vGLUT1 and vGLUT2 terminals were mapped on a montage of the low-power images of the thalamus and the camera lucida drawings by screening the whole sections with the $40 \times$ and $63 \times$ objectives. The maps were finally matched with the corresponding stereotaxic level. Neighboring vGLUT1- and vGLUT2-immunostained sections were mapped at $500 \mu \mathrm{m}$ intervals. Only large $(>2 \mu \mathrm{m})$, clearly distinguishable vGLUT1- or vGLUT2-immunoreactive terminals were considered for the initial light microscopic mapping. The borders drawn with the light microscope were verified using electron microscopy (see below). Finally, a composite map was drawn using the individual maps of the animals.

At the electron microscopic level, terminals of a morphological type known as RL ( $\mathrm{R}$ for round vesicles and $\mathrm{L}$ for large terminal size) were defined using the following criteria: large size $(>2 \mu \mathrm{m}$ of diameter), round vesicles, multiple active zones, multiple puncta adherentia (also called filamentous contacts), multiple mitochondria cross-sections, large-caliber dendrite or vesicle-filled dendrites as postsynaptic targets, glial ensheathment. Our aim was to compare the size of RL terminals in different nuclei. Proper 3D reconstruction of a statistically meaningful number of terminals using serial electron microscopic images from a large number of thalamic nuclei was implausible. Thus, after careful consideration of the 3D-reconstructed terminals in the literature (Mason et al., 1996; Beggs et al., 2003; Budisantoso et al., 2012), our own previous experience with large thalamic terminals (Barthó et al., 2002; Bokor et al., 2005; Lavallée et al., 2005; Bodor et al., 2008; Wanaverbecq et al., 2008), and the present material, we decided to measure the cross-sectional area of a large number of terminals on single sections $(n=695)$ as a best proxy for terminal size. To compare the size of RL terminals in different nuclei, the following criteria were used for the measurements: the terminals should contain multiple active zones and/or puncta adherentia and at least four mitochondria. Based on previous data, multiple mitochondria, active zones, or puncta adherentia are present at or close to the largest cross-sectional area of a terminal reconstructed in 3D from serial sections. Thus, in this way, we minimized the error introduced by sampling $\mathrm{RL}$ terminals close to their ends and also avoided sampling RS ( $\mathrm{R}$ for round vesicles and $\mathrm{S}$ for small terminal size) terminals. RL terminals meeting our criteria were outlined, and their cross-sectional areas were measured by NIH ImageJ.

Statistics. We tested the normality of the sample distributions in case of the axon terminals with the Shapiro-Wilk $W$ test, and, because of the significant values in every group, we applied nonparametric statistics (Kruskal-Wallis test, multiple comparison). Because it was significant, we compared the different groups with the Bonferroni's-corrected twotailed test using the Statistica software.

\section{Results}

In the present account, the nomenclature of Paxinos et al. (2000) is applied for the thalamic nuclei. Because there is a considerable confusion regarding the proper name and exact nuclear division of the primate thalamus, it is important to emphasize that the core concept of the results does not depend on the nomenclature used.

\section{vGLUT1 and vGLUT2 as markers for cortical and subcortical terminals}

vGLUT1 and vGLUT2 are widely used to selectively label cortical and subcortical terminals, respectively (Lavallée et al., 2005; Graziano et al., 2008; Masterson et al., 2009). To establish whether these markers are applicable in our conditions, we made the following observations and experiments.

\section{$v$ GLUT1 and $v$ GLUT2 colocalization}

To establish distinct origins, colocalization of the two markers was performed at the level of thalamic axon terminals using two different methods: postembedding double immunofluorescence (Fig. $1 A-C$ ) and preembedding double labeling using two different chromogens (DAB and silver-intensified gold particles) at the electron microscopic level (see Figs. 4 and 8). None of these methods revealed colocalization between the two markers.

\section{Combination of tract tracing and immunocytochemistry}

In three animals, cortical terminals were labeled by the anterograde tracer BDA. The distribution of large cortical terminals was consistent with the distribution of vGLUT1-positive terminals, and colocalization studies demonstrated that large cortical terminals are vGLUT1 immunoreactive (Fig. $1 D-F$ ).

In addition to this, the general distribution of vGLUT1- and vGLUT2-positive terminals in the thalamus and the surrounding brain regions was entirely consistent with previous tract-tracing data (for details, see Results and Discussion).

\section{Large vGLUT2-immunoreactive terminals in the thalamus}

Immunostaining for vGLUT2 with DAB-Ni labeled large (2-7 $\mu \mathrm{m}$ ) irregularly shaped structures in several thalamic nuclei (Fig. $2 A)$. Electron microscopic samples obtained from the lateral geniculate nucleus (LGN) and the ventral posterolateral and posteromedial nucleus (VPL/VPM) using the preembedding gold method demonstrated that vGLUT2-positive elements were large axon terminals that contained several mitochondria and established multiple synapses and puncta adherentia on their postsynaptic targets (Fig. 2B). Innervation of vesicle-filled dendrites was also observed. These features are compatible with wellcharacterized RL-type driver terminals in the dorsal thalamus (Colonnier and Guillery, 1964). The area of the terminals was measured in single electron microscopic sections (see Materials and Methods). Pooled terminal sizes were not distributed normally (Shapiro-Wilk test, $W=0.941, p=0.0264$ for LGN and 

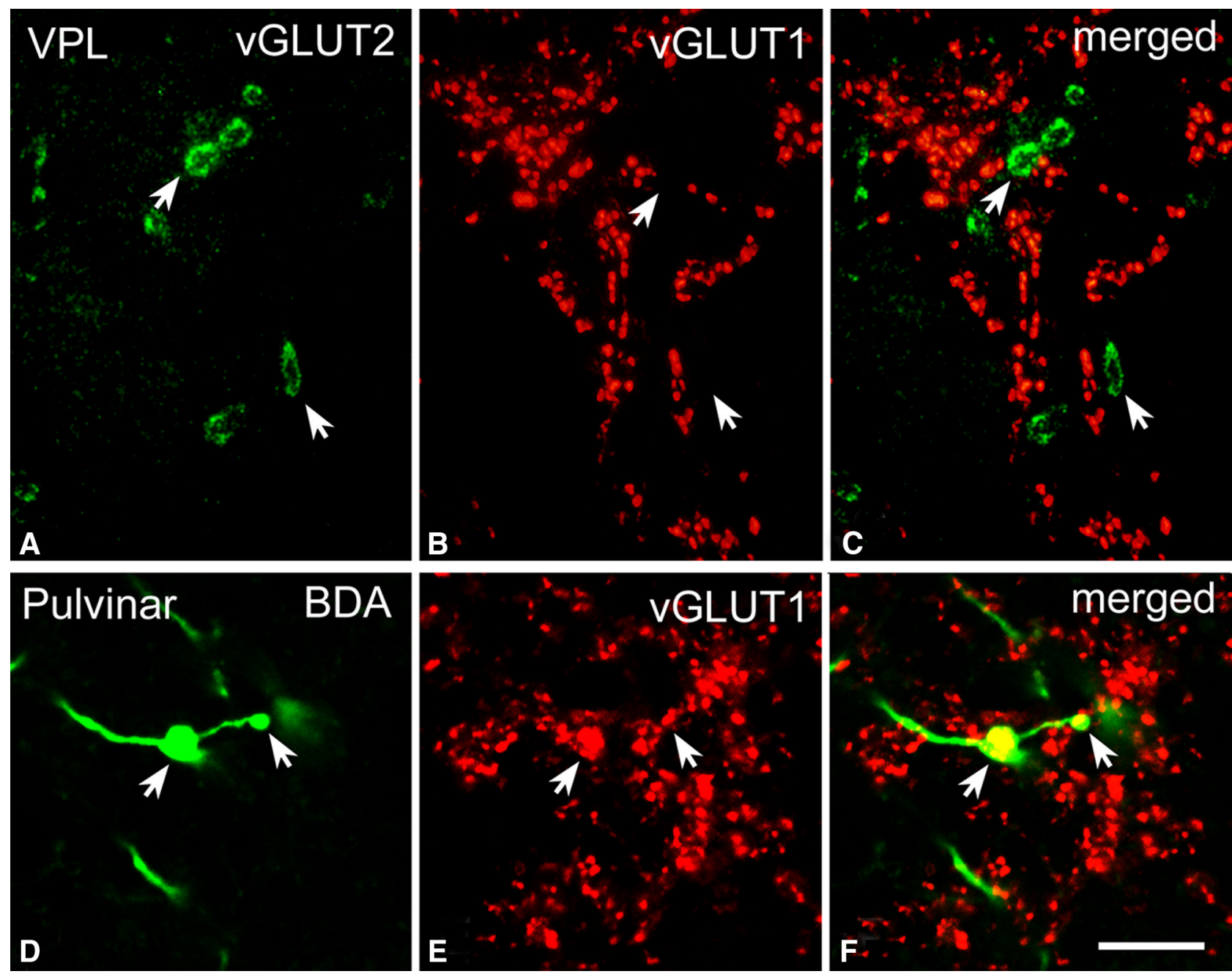

Figure 1. High-power fluorescent images of vGLUT1- and vGLUT2-immunoreactive terminals. $\boldsymbol{A}-\boldsymbol{C}$, High-power fluorescent photomicrographs demonstrating that vGLUT2 (A) and vGLUT1 ( $\boldsymbol{B}$ ) label distinct sets of afferents in the ventral posterolateral nucleus of the macaque monkey. $\boldsymbol{D}-\boldsymbol{F}$, High-power fluorescent photomicrographs demonstrating that anterogradely labeled large cortical terminals (green, arrows in $\boldsymbol{D}$ ) display vGLUT1 immunoreactivity $($ red, $\boldsymbol{E})$ in the pulvinar. Scale bar, $10 \mu \mathrm{m}$.

$W=0.821, p=0.000001$ for VPL) and showed a skewed distribution toward larger values. The area of RL terminals were large in both nuclei and did not differ significantly (LGN median, 6.02 $\mu \mathrm{m}^{2}$; first to third quartile range, 4.63-8.46 $\mu \mathrm{m}^{2} ; n=44$; VPL median, $4.45 \mu \mathrm{m}^{2}$; first to third quartile range, $3.20-6.12 \mu \mathrm{m}^{2}$; $n=116 ; p=0.913$ ).

Large vGLUT2-immunoreactive terminals were distributed unevenly in the thalamus (Fig. 2C). Dense vGLUT2-positive innervation characterized the major primary sensory relay nuclei, including the LGN, VPL/VPM, and the anterior portion of the medial geniculate nucleus, in agreement with the retinal (Colonnier and Guillery, 1964; Wilson, 1989), spinal trigeminal (Boivie, 1979; Rausell et al., 1992), and inferior collicular (Molinari et al., 1995) sources of their driver inputs, respectively (Fig. 2C). The dense vGLUT2-immunoreactive innervation continued rostrally from the VPL/VPM complex and extended to the entire ventrolateral nucleus (VL), which is consistent with its cerebellar inputs (Rouiller et al., 1994; Percheron et al., 1996). The immunostaining became patchy farther rostrally as the cerebello-recipient motor thalamus (VL) merged into the basal ganglia-recipient ventral anterior nucleus (VA), as described previously (Bodor et al., 2008). In the anterior portion of the thalamus, the anterior nuclear group displayed dense vGLUT2 immunostaining, consis- tent with subcortical driver inputs from the mammillary body described $>200$ years ago (Vicq d'Azyr, 1786) (Fig. 2C).

To examine whether all RL-type axon terminals express vGLUT2 in these regions, electron microscopic samples from the VPL and LGN were systematically screened after preembedding gold immunostaining. In our sample, each RL terminal encountered was immunoreactive for vGLUT2 $(n=160)$. These data also demonstrate that false-negative RL terminals do not confound our results. Conversely, after vGLUT1 immunostaining of the same nuclei in neighboring sections, no RL terminals were vGLUT1 immunoreactive.

Interestingly, large vGLUT2-immunoreactive terminals were abundant in several nuclei that are known to receive driver inputs from the neocortex and are considered as higher-order nuclei (Fig. 2C). These included the medial region of the mediodorsal nucleus together with the adjacent midline nuclei, the rostral intralaminar nuclei with the paralamellar part of the mediodorsal nucleus, and parts of the anterior and lateral pulvinar. Conversely, we found large thalamic territories that were devoid of any vGLUT2-immunoreactive inputs (Fig. 2C).

These data demonstrate that large thalamic territories are innervated only by subcortical RL-type terminals and receive driver inputs from no other sources. In addition, certain zones of 

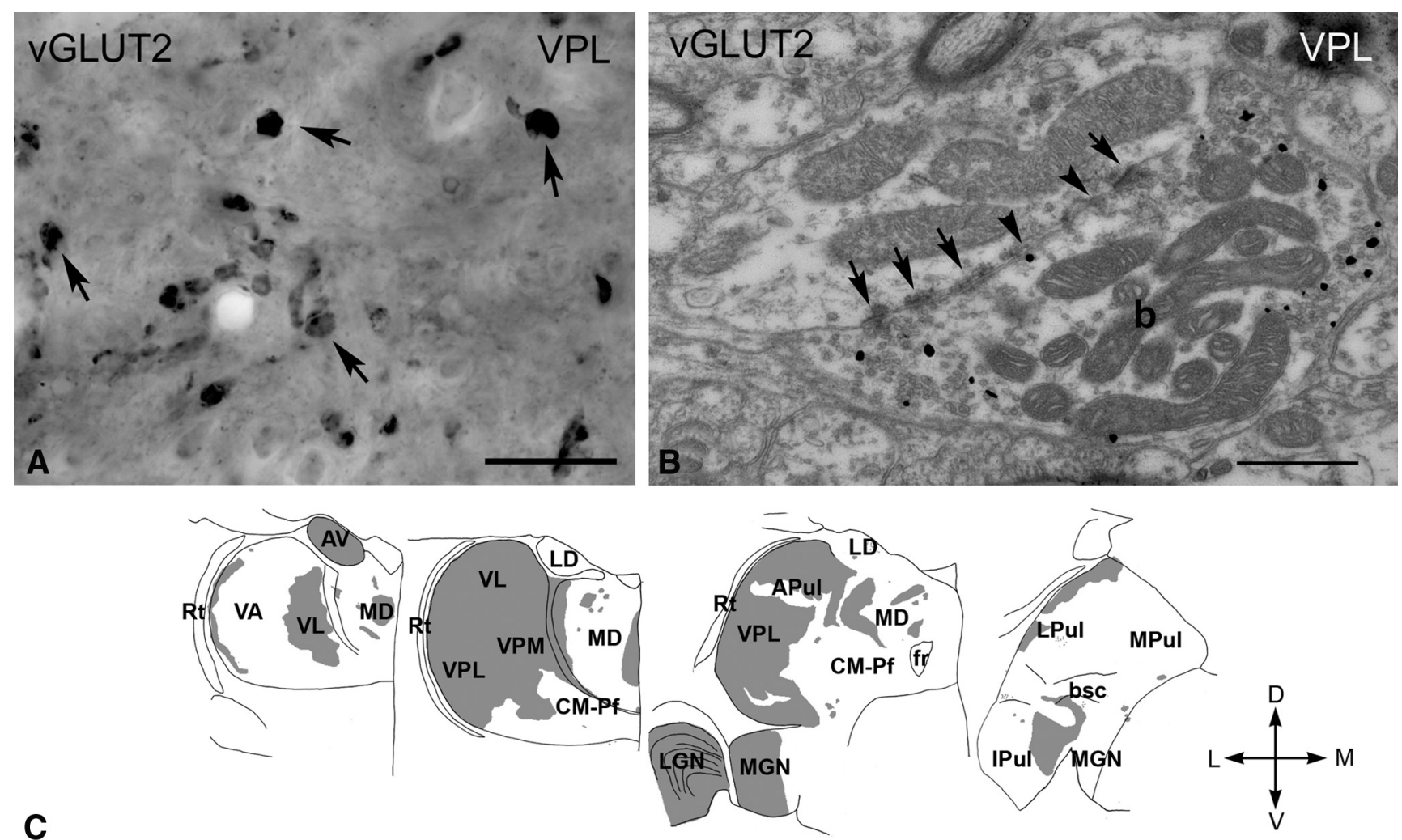

Figure 2. VGLUT2 -immunoreactive terminals in the macaque thalamus. A, High-power light microscopic image of a vGLUT2-immunostained section using DAB-Ni as a chromogen from the VPL. VGLUT2 labels large irregularly shaped terminals (arrows). B, High-power electron micrograph of a vGLUT2-positive terminal (b) labeled by silver intensified gold particles (small black dots). The terminal displays the ultrastructural features of RL-type terminals, including large size, multiple mitochondria, puncta adherentia (arrowheads), synapses (arrows). C, Regional distribution of large VGLUT2-positive terminals at four coronal levels of the macaque thalamus arranged from rostral to caudal levels. Note the absence of subcortical driver inputs from large thalamic territories. Scale bars: $A, 20 \mu \mathrm{m} ; \boldsymbol{B}, 0.5 \mu \mathrm{m}$. APul, Anterior pulvinar; AV, anteroventral; bsc, brachium of superior colliculus; CM-Pf, centromedian-parafascicular; fr, fasciculus retroflexus; Ipul, inferior pulvinar; LD, laterodorsal; LGN, lateral geniculate nucleus; LPul, lateral pulvinar; MD, mediodorsal; MGN, medial geniculate nucleus; MPul, medial pulvinar; VA, ventral anterior; VL, ventrolateral; VPL, ventral posterolateral; VPM, ventral posteromedial; Rt, reticular thalamus.

higher-order thalamic nuclei also receive substantial subcortical inputs. Finally, significant thalamic territories are completely devoid of any large subcortical glutamatergic terminal and hence are free from major subcortical influence.

\section{Large vGLUT1-immunoreactive terminals in the thalamus}

At the light microscopic level, small and large vGLUT1immunoreactive terminals could be distinguished corresponding to the dual cortical innervation (layer 6 and layer 5, respectively) of the thalamus (Rouiller and Welker, 2000) using DAB-Ni as a chromogen. The small $(<1 \mu \mathrm{m}$ diameter) terminals densely filled the entire thalamus (Fig. $3 \mathrm{~A}, \mathrm{C}$ ). At the electron microscopic level after preembedding gold immunostaining, small vGLUT1-positive terminals targeted thin dendrites with a single synapse, lacked puncta adherentia, and rarely contained mitochondria (Fig. 3B). This is consistent with the ultrastructural features of RS-type terminals known to originate from layer 6 (Jones and Powell, 1969; Ralston and Herman, 1969). The area of the small terminals were an order of magnitude smaller than those of the RL types (median, $0.47 \mu \mathrm{m}^{2}$; first to third quartile range, $0.19-1.09 \mu \mathrm{m}^{2} ; n=58$ measured in VPL; $p<0.000001$ ).

At the light microscopic level, large thalamic territories contained only small vGLUT1-positive terminals (Fig. $3 A, B$ ). However, in several regions clearly distinguishable, giant vGLUT1-containing terminals were also present in addition to the small ones (Fig. 3C,D). Giant vGLUT1-positive terminals were distributed mainly in the mediodorsal nucleus and the pulv- inar (Fig. 3E), consistent with tract tracing studies demonstrating dual corticothalamic innervation of these nuclei (Schwartz et al., 1991; Taktakishvili et al., 2002; Cappe et al., 2007). In addition, a small territory receiving large vGLUT1 terminals was observed ventrally to VL and the somatosensory VPM/VPL. According to some accounts, this region is selectively termed as ventral posterior inferior nucleus (VPI) (Jones, 2007) and is thought to be involved in pain sensation (Apkarian and Shi, 1994).

Electron microscopic observations from the lateral pulvinar demonstrated that the large vGLUT1-immunoreactive endings displayed the ultrastructural features of RL terminals and replicated the morphological properties of vGLUT2-positive terminals (large size, large-caliber target, multiple synapses, puncta adherentia and mitochondria, triadic arrangement; Fig. 3D). These features are compatible with the cortical layer 5 origin of large vGLUT1 terminals (Deschênes et al., 1994; Bourassa and Deschênes, 1995; Bourassa et al., 1995).

The area of terminals in the lateral pulvinar (median, 4.56 $\mu \mathrm{m}^{2}$; first to third quartile range, $3.34-5.75 \mu \mathrm{m}^{2} ; n=64$ ) measured in single electron microscopic sections were not significantly different from drivers labeled by vGLUT2 in VPL or in the LGN (Bonferroni's-corrected two-sided test, $p=1$ for both nuclei).

To examine whether all RL terminals express vGLUT1, electron microscopic samples from the lateral pulvinar were systematically screened after preembedding gold immunostaining. Sixty-four of 66 RL-type nerve endings were immunoreactive for 

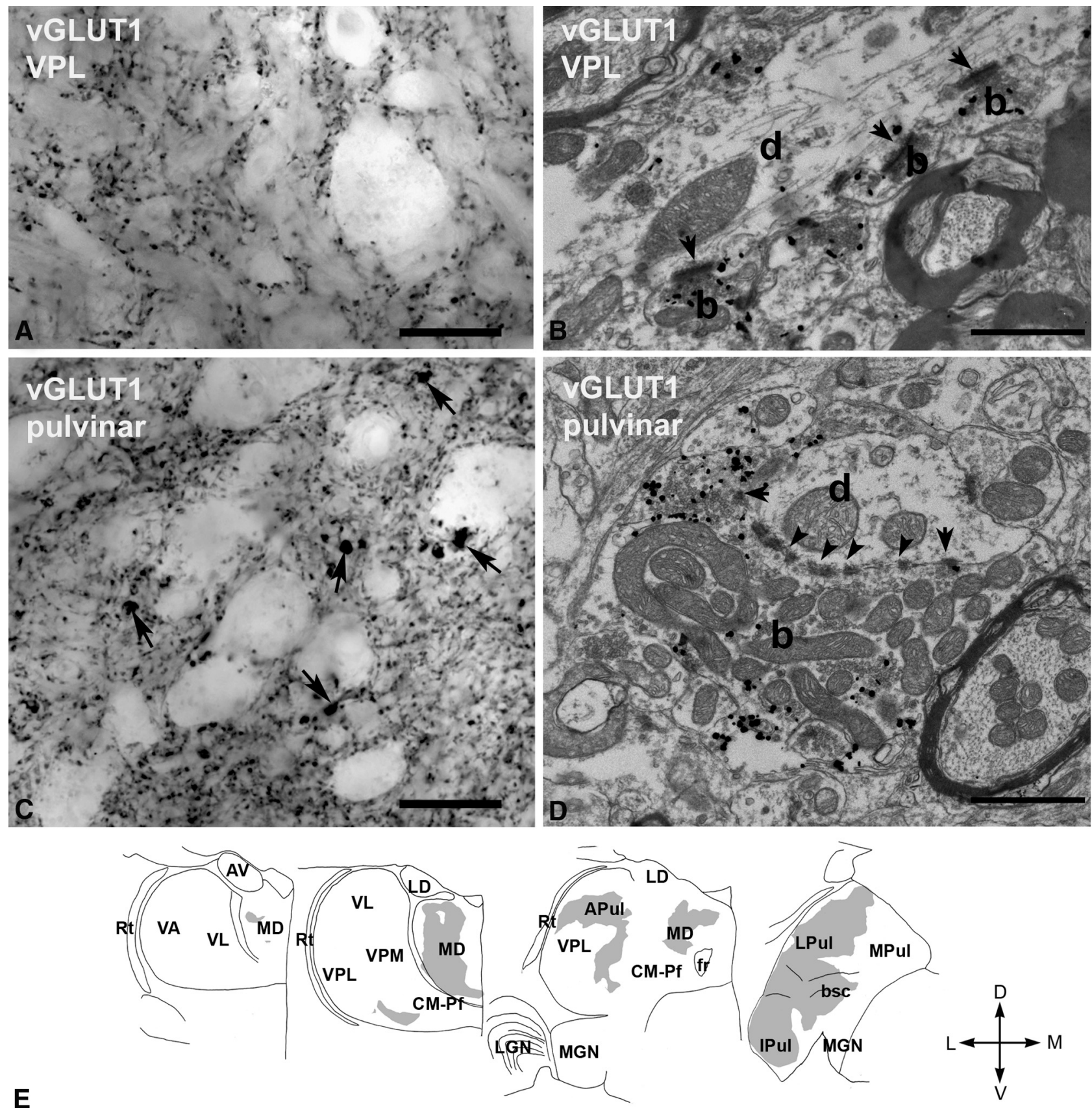

Figure 3. vGLUT1-immunoreactive terminals in the macaque thalamus. A, High-power light microscopic image of a vGLUT1-immunostained section from the VPL displaying small vGLUT1immunoreactive terminals only. $\boldsymbol{B}$, At the electron microscopic level, these terminals (b, silver-intensified gold) display the features of RS-type terminals, i.e., small size, maximum of one or two mitochodria, no puctum adherens, and a single synapse (arrow). d, Postsynaptic dendrite. $C$, In the lateral pulvinar, besides the small vGLUT1-immunoreactive terminals, large immunoreactive structures (arrows) can also be distinguished at the light microscopic level. $\boldsymbol{D}$, At the electron microscopic level, a large vGLUT1-immunoreactive terminal (b, silver-intensified gold) displays all the ultrastructural features of RL terminals. Arrows, Synapses; arrowheads, puncta adherentia; d, postsynaptic dendrite. E, Regional distribution of large vGLUT1-positive terminals at four coronal levels of the macaque thalamus. Note the lack of large vGLUT1-positve terminals from many thalamic nuclei considered as higher order and the heterogeneity of the distribution within the mediodorsal nucleus (MD) and pulvinar. Scale bars: $A, C, 20 \mu \mathrm{m} ; \boldsymbol{B}, \boldsymbol{D}, 1 \mu \mathrm{m}$. APul, Anterior pulvinar; AV, anteroventral; bsc, brachium of superior colliculus; CM-Pf, centromedian-parafascicular; fr, fasciculus retroflexus; Ipul, inferior pulvinar; LD, laterodorsal; LGN, lateral geniculate nucleus; LPul, lateral pulvinar; MD, mediodorsal; MGN, medial geniculate nucleus; MPul, medial pulvinar; VA, ventral anterior; VL, ventrolateral; VPL, ventral posterolateral; VPM, ventral posteromedial; Rt, reticular thalamus.

vGLUT1, demonstrating that virtually no other type of large excitatory inputs innervate these regions. These data again demonstrate that false-negative RL terminals do not confound our results.

Large vGLUT1-positive terminals were not distributed uniformly in the mediodorsal nucleus and pulvinar (Fig. 3E). More specifically, they were found only in the central part of the medi- odorsal nucleus, because its medial and lateral aspects were devoid of these terminals. In the case of the pulvinar, vGLUT1immunoreactive drivers were observed in the anterior, lateral, and inferior portion of the nucleus but not in its medial part. In several nuclei that are traditionally regarded as higher order [e.g., intralaminar nuclei, centromedian-parafascicular (CMPf) complex], no large vGLUT1 terminals could be discerned 

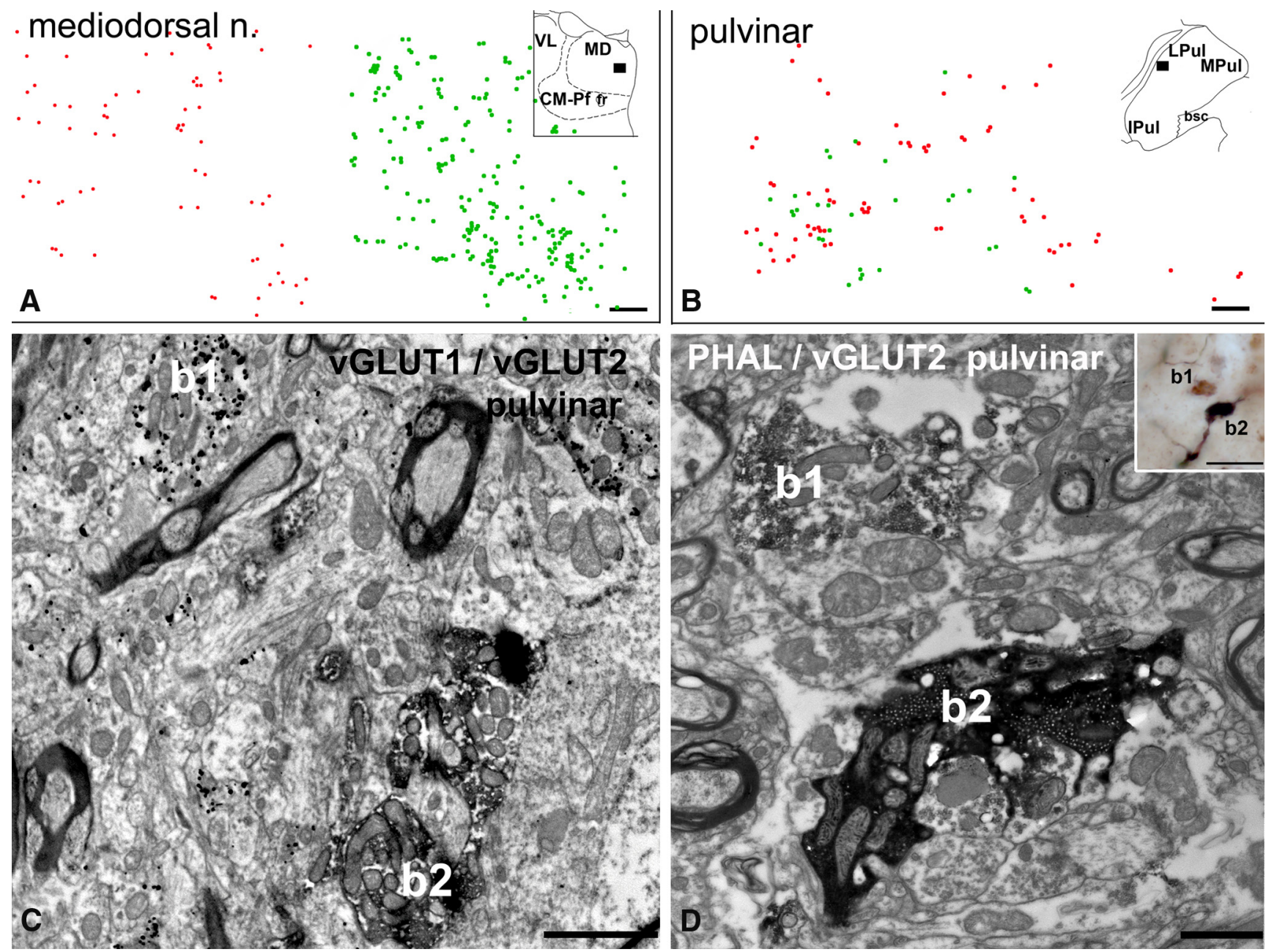

Figure 4. Convergence of large vGLUT1 and vGLUT2 terminals. $\boldsymbol{A}, \boldsymbol{B}$, Plot of large vGLUT1-immunoreactive (red dots) and vGLUT2-immunoreactive (green dots) terminals using doubleimmunostained material in the mediodorsal nucleus $(\boldsymbol{A})$ and pulvinar ( $\boldsymbol{B}$, position shown in the inset). Note clear separation of the two types of terminals in the mediodorsal nucleus and homogeneous mixing in the lateral pulvinar. $C$, Electron micrograph from the anterior pulvinar displaying close proximity $(<3 \mu \mathrm{m})$ of a large vGLUT1-immunoreactive terminal (b1, silverintensified gold particles) and a large vGLUT2-positive terminal (b2, DAB precipitate). D, Correlated light (inset) and electron micrographs in the anterior pulvinar depicting a vGLUT2immunoreactive large terminal (b1, brown DAB precipitate) adjacent to a large cortical terminal (b2, black DAB-Ni precipitate) anterogradely labeled from the supplementary motor cortex by Phaseolus vulgaris leucoagglutinin (PHAL). Both terminals display RL features. Scale bars: $A, B, 50 \mu \mathrm{m} ; C, 2 \mu \mathrm{m} ; \mathbf{D}, 1 \mu \mathrm{m}$; inset, $20 \mu \mathrm{m}$.

at the light microscopic level (for additional analysis of these nuclei, see below).

The above data demonstrate that large thalamic territories in the pulvinar and mediodorsal nucleus are entirely under the control of cortical drivers and that they receive large excitatory terminals from no other sources. However, both the pulvinar and the mediodorsal nucleus appear to be heterogeneous and contain a mosaic of driver inputs with different origin.

\section{Convergence of large vGLUT1- and}

\section{vGLUT2-posititve terminals}

The initial screen of our material indicated that the distribution of vGLUT1- and vGLUT2-immunostained giant terminals is mostly complementary (non-overlapping) in the primate thalamus. However, careful examination revealed regional codistribution of vGLUT1- and vGLUT2-positive terminals along the borders of territories innervated by subcortical and cortical drivers. These zones included the borders of mediodorsal and centrolateral nuclei, the transition between VPI and VPM, and the border regions of the ventrolateral nucleus with the anterior or lateral pulvinar. Convergent zones were also observed at the medial aspect of the inferior pulvinar (for a complete map, see Fig. 9).

To examine the codistribution of large vGLUT1- and vGLUT2-positive terminals, we performed double immunostaining for vGLUT1 and vGLUT2 using DAB and DAB-Ni as chromogens. Two different patterns were observed. In the medial part of the mediodorsal nucleus, clear segregation of the cortical and subcortical drivers was detected (Fig. 4A). However, in the lateral pulvinar, homogeneous mixing of vGLUT1- and vGLUT2-immunoreactive large terminals was found (Fig. $4 B$ ). Electron microscopic analysis of convergent zones from the anterior pulvinar after double immunostaining for the two markers (DAB and preembedding gold) confirmed that both vGLUT1and vGLUT2-immunoreactive large terminals display driver (RL-type) features and can be found in close proximity $(<5 \mu \mathrm{m})$ to each other in single electron microscopic sections (Fig. 4C). Direct demonstration of the convergence of two different drivers on the same relay cell was not attempted in this analysis.

These data were confirmed by using anterograde tracing of the cortical terminals ( $n=3$ monkeys). Cortical injections (for details, see Materials and Methods) labeled small and large ter- 

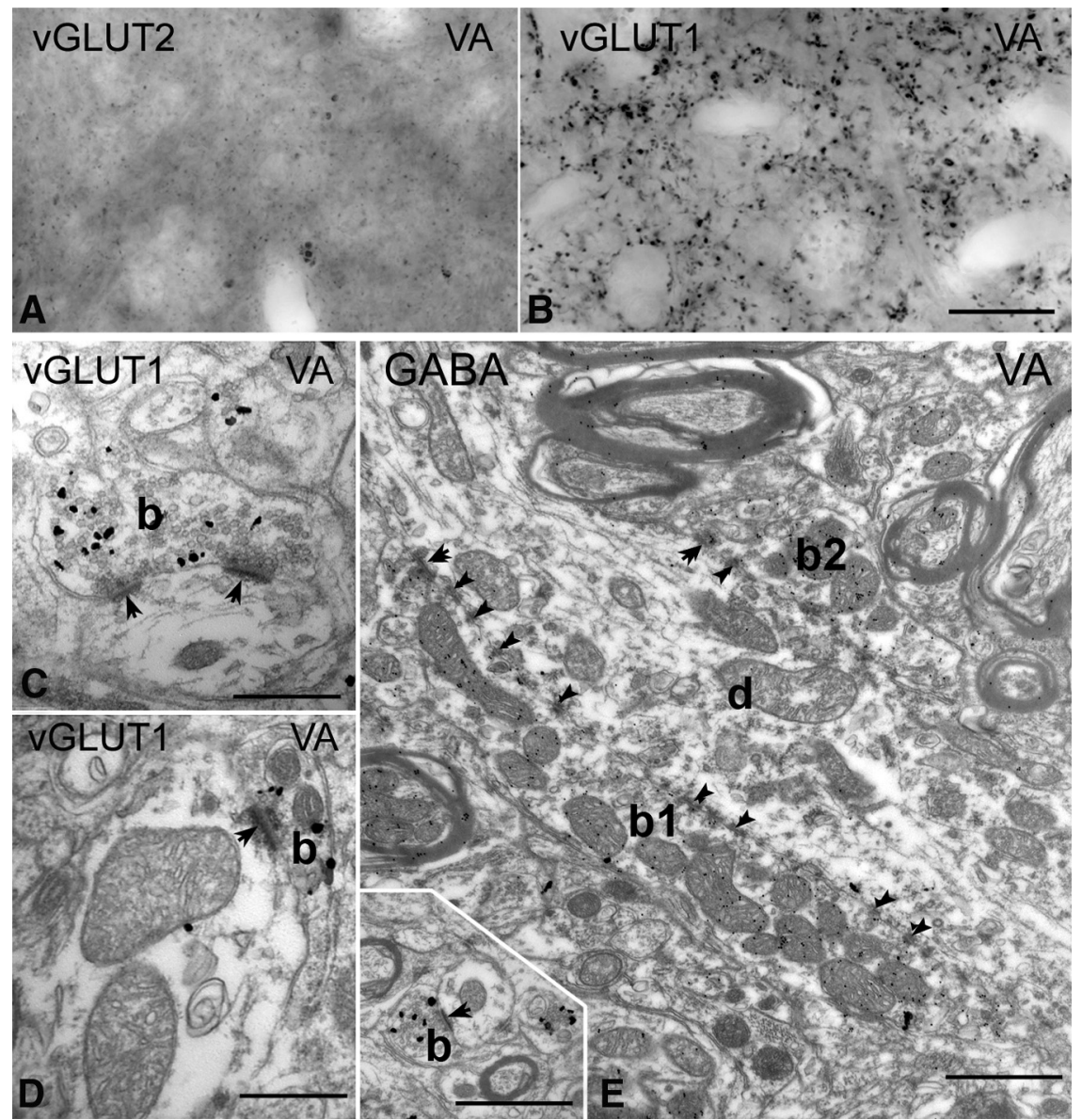

Figure 5. Thalamic territories without large vGLUT1 or vGLUT2 terminals. $A, B$, High-power light microscopic images of vGLUT2-immunostained $(\boldsymbol{A})$ and vGLUT1-immunostained $(\boldsymbol{B})$ sections from the ventral anterior nucleus display the lack of large terminal labeling by both markers. $C, D$, At the electron microscopic level, vGLUT1-positive terminals show the features of RS-type terminals. Most of them form a single synapse or more rarely two synapses and contained a maximum of two mitochondria. $\boldsymbol{E}$, The large terminals of the ventral anterior nucleus (b1 and b2) are negative for vGLUT1 but show GABA immunoreactivity after postembedding GABA immunogold staining. Inset shows a vGLUT1-immunoreactive terminal at the same magnification. Scale bars: $A, B, 20 \mu \mathrm{m} ; \boldsymbol{C}, \boldsymbol{D}, 500 \mathrm{~nm} ; \boldsymbol{E}$ and inset, $1 \mu \mathrm{m}$.

minals in the thalamus. Because the injections involved motor cortical areas, small terminals were mainly concentrated in the ventrolateral nucleus. A similar pattern was observed after tracer injection into the ventral part of the internal capsule (see Materials and Methods). Large BDA-labeled terminals were only found in regions in which vGLUT1-immunoreactive terminals have also been observed (mediodorsal nucleus and pulvinar). Direct colocalization using double immunofluorescence demonstrated that large cortical terminals express vGLUT1 (Fig. 1). In two of three animals, some of the labeled large cortical terminals were found intermingled with large vGLUT2-positive terminals, whereas the rest were found in vGLUT2-free regions. Double staining for the tracer and vGLUT2 demonstrated anterogradely labeled cortical terminals in close proximity to vGLUT2-positve terminals at both the light and electron microscopic levels (Fig. 4D).

These data confirm that well-defined thalamic territories can be under the control of both cortical and subcortical driver afferents.

\section{Lack of large excitatory terminals}

After mapping the distribution of large vGLUT1 and vGLUT2 terminals, at the light microscopic level significant thalamic ter- ritories $(\sim 35 \%)$ appeared to lack both types of large excitatory terminals. The failure to detect giant terminals can be the result of (1) the presence of large excitatory inputs not labeled by vGLUTs, (2) the genuine lack of large excitatory inputs, or (3) the technical limit of light microscopic resolution. To examine these possibilities, we performed electron microscopic analysis in thalamic nuclei apparently lacking drivers.

In the ventral anterior nucleus, known to receive subcortical inputs from the basal ganglia (Ilinsky et al., 1985; Rouiller et al., 1994), immunostaining for vGLUT2 revealed no immunoreactive structure (Fig. $5 A$ ), whereas vGLUT1 immunostaining labeled only small terminals (Fig. $5 B$ ). Confirming the light microscopic results, even after extensive searches, no vGLUT1immunoreactive RL terminal could be observed at the electron microscopic level. All vGLUT1-positive terminals in VA were RS type and thus were small, established at most two synaptic contacts, rarely contained more than one mitochondrial crosssection, and lacked puncta adherentia (Fig. $5 C, D$, inset on $E$ ). We compared the size of vGLUT1-immunoreactive terminals in VA with vGLUT1 terminals in VPL, which has only RS-type vGLUT1 input. The area of vGLUT1-immunoreactive terminals (median, $0.40 \mu \mathrm{m}^{2}$; range, $0.30-0.55 \mu \mathrm{m}^{2}$; $n=83$ ) were not statistically different (Bonferroni's-corrected two-sided test, $p=$ 1) from the vGLUT1 terminals of VPL, in which only layer 6 input is present (median, $0.43 \mu \mathrm{m}^{2}$; first to third quartile range, $0.29-$ $0.56 \mu \mathrm{m}^{2} ; n=58$ from the VPL).

However, the neuropil of the ventral anterior nucleus contained large, multisynaptic, vGLUT1immunonegative terminals that innervated thick proximal dendrites in great numbers. Because this area is known to be innervated by GABAergic basal ganglia afferents that form large inhibitory terminals (Kultas-Ilinsky and Ilinsky, 1990; KultasIlinsky et al., 1997; Bodor et al., 2008), we replicated the experiments after postembedding immunostaining for GABA. In our sample, all large terminals displaying multiple synapses, puncta adherentia, and mitochondria displayed GABA immunoreactivity $(n=20$; Fig. $5 E)$.

These data demonstrate that basal ganglia-recipient nuclei contain no giant excitatory driver input. This input is replaced by large GABAergic afferents in the proximal dendritic domains of TC cells.

\section{Heterogeneity of multisynaptic RL terminals}

Beside the ventral anterior nucleus, other thalamic regions also lacked a discernible number of large terminals at the light microscopic level. These included the medial pulvinar and the laterodorsal nucleus (Fig. 6).

In the medial pulvinar, in contrast to the apparent homogeneity of vGLUT1-immunostaining at the light microscopic level, two types of vGLUT1-immunoreactive nerve endings could be 
readily distinguished at the electron microscopic level. The majority of vGLUT1 terminals were similar to the small vGLUT1 terminals of the other nuclei examined (no or single mitochondria, no puncta adherentia, single synapse onto a thin caliber dendrite). In addition to these boutons, however, larger terminals with RL-type features (multiple mitochondria, multiple active zones or puncta adherentia) were also observed (Fig. 6B). The cross-sectional area of RL-type medial pulvinar terminals (median, $2.24 \mu^{2}$; first to third quartile range, $1.64-2.83$ $\left.\mu \mathrm{m}^{2} ; n=50\right)$ was less than half of that of vGLUT1-immunoreactive RL terminals in the lateral pulvinar or vGLUT2positive RL-type terminals in the VPL or LGN. As a consequence, the size of RL terminals in the medial pulvinar versus the size of RL terminals in the other three nuclei examined were highly significantly different ( $p<0.00001$ in all cases) (Fig. $7 A)$. Similarly, multisynaptic vGLUT1immunoreactive terminals of the laterodorsal nucleus were also considerably smaller than their counterparts in the lateral pulvinar (median, $1.76 \mu \mathrm{m}^{2}$; first to third quartile range, $0.97-2.27 \mu \mathrm{m}^{2} ; n=$ $35 ; p<0.00001$; Figs. $6 D, 7 A$ ).

These data demonstrate that there is a considerable nucleus-specific variability in the size of vGLUT1-positive RLtype terminals. These small RL terminals cannot be resolved at the light microscopic level because of the abundance of RS terminals but clearly display RL features at the electron microscopic level.

The size of vGLUT2 RL-type terminals also displayed significant variability and nuclear specificity. vGLUT2-immunoreactive terminals in the medial part of the mediodorsal nucleus were half the size of vGLUT2 terminals in the LGN (median, 2.78 $\mu \mathrm{m}^{2}$; range, $2.16-3.91 \mu \mathrm{m}^{2} ; p<0.001 ; n=44$; Fig. $7 B$ ). In addition, the laterodorsal nucleus also contained a small number of small vGLUT2 terminals in this size range that were significantly smaller than the drivers in the LGN or VPL (median, 2.02 $\mu \mathrm{m}^{2}$; first to third quartile range, $1.42-3.00 \mu \mathrm{m}^{2} ; n=12 ; p<$ 0.001 for LGN and $p<0.05$ for VPL).

These data indicate that the size and complexity of RL terminals are highly variable in the primate thalamus and display significant regional/nuclear differences.

\section{Intralaminar nuclei}

An important nuclear group in which large vGLUT1immunoreactive terminals were not observed at the light microscopic level is the intralaminar nuclei. Given the strategic importance of these nuclei in consciousness (Castaigne et al., 1981; Laureys et al., 2000; Schiff et al., 2007; Yamamoto et al., 2010) and their designation as higher-order nuclei, we specifically aimed to determine their driver inputs.

The intralaminar nuclei consist of two parts: the caudal CM-Pf complex and the rostral intralaminar nuclei. In the case of the CM-Pf complex, vGLUT1-postive RL-type terminals were exceedingly rare. Even after extensive searches, only nine vGLUT1-positive RL-type terminals were found that fulfilled our criteria (see Materials and Methods). The size of these terminals (median, $1.78 \mu \mathrm{m}^{2}$; first to third quartile range, $1.29-2.06 \mu \mathrm{m}^{2}$ ) were in the range of small RL-type terminals encountered in the medial pulvinar and the laterodorsal nucleus (Fig. 7A). Large multisynaptic terminals were immunoreactive for GABA, as in the case of the ventral anterior nucleus, consistent with the basal ganglia inputs of the CM-Pf complex (KultasIlinsky et al., 1997).

The rostral intralaminar nuclei are a thin sheet of cells that surround the mediodorsal nucleus. The borders of the intralaminar nuclei cannot be unequivocally determined using vGLUT1- or vGLUT2-immunostained sections. Thus, we used calbindin-D28 immunostaining, a well-characterized marker of the rostral intralaminar group (Jones, 2007), to exactly define the borders. Next, we prepared adjacent sections double immunostained for vGLUT1 and vGLUT2 and selected the appropriate area using capillaries as landmarks (Fig. $8 A-G$ ).

In the rostral intralaminar group, the vGLUT1-positive terminals displayed almost exclusively RS features. Even after an extensive search, only two vGLUT1-positive terminals were found that fulfilled our criteria for RL terminals. The same region contained vGLUT2-immunoreactive RL terminals, which were smaller than the large vGLUT2 terminals of VPL and LGN but were comparable in size to vGLUT2 terminals of the medial part of the mediodorsal nucleus (median, $2.82 \mu \mathrm{m}^{2}$; first to third quartile range, $2.15-4.32 \mu \mathrm{m}^{2} ; n=49$; Fig. $7 B$ ). The presence of subcortical driver inputs in the rostral intralaminar nuclei is consistent with the literature showing afferents arising from the superior colliculus (Harting et al., 1980) and the spinal cord (Apkarian and Shi, 1994).

The data indicate that intralaminar nuclei have few or no multisynaptic inputs from the cortex, but the rostral intralaminar group is innervated by subcortical drivers of medium size. 
A

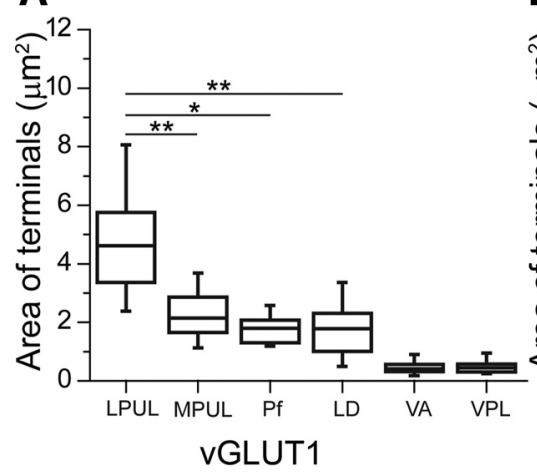

B

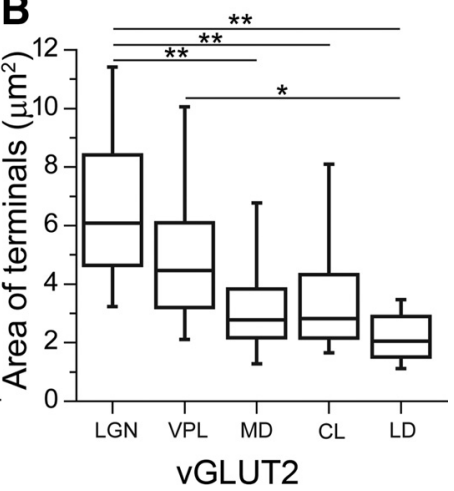

The data can serve as a framework to explain primate behavioral results and can be used to understand the morphological bases of human neurological diseases involving the thalamus (see below).

\section{Technical considerations}

vGLUT1 and vGLUT2 were treated in this paper as selective markers for cortical and subcortical terminals, respectively. Our present data and a review of the recent literature confirm this distinction. The mapping of vGLUT1 and vGLUT2 terminals in this study is entirely consistent with previous track tracing data. First, in nuclei with known subcortical input (cerebellum, spinal cord, trigeminal complex, retina, brainstem, mammillary body), only large vGLUT2 terminals were found, making it unlikely that any thalamicprojecting subcortical center expresses significant amount of vGLUT1 (for details, see Results and below). Second, large

\section{The map of drivers in the primate thalamus}

Based on the light and electron microscopic results, we prepared a complete map of driver distribution in the primate thalamus (Fig. 9). The map indicates the origin (cortical vs subcortical), the size (large, medium, or no drivers), as well the convergence or segregation of drivers with different origin. Based on the exact composition of driver assembly, six different thalamic territories could be distinguished: (1) regions innervated exclusively by large vGLUT2 terminals, (2) regions innervated exclusively by large vGLUT1 terminals, (3) regions receiving both vGLUT1- and vGLUT2-positive large terminals, (4) regions without RL terminals of any type, (5) regions innervated by small RL-type vGLUT1 terminals, and (6) regions with small vGLUT1- and small vGLUT2-positive RL type terminals.

These territories primarily but not entirely overlapped with previously established nuclear borders (Fig. 9). vGLUT1 and vGLUT2 immunostainings were suitable to delineate thalamic territories with significant clinical relevance. Large vGLUT2 terminals delineated the borders of cerebello-recipient thalamus and basal ganglia-recipient thalamic territories. The different driver composition enabled us to identify the borders between the pulvinar and the surrounding sensory and motor regions. Conversely, the similar driver composition did not allow us to distinguish somatosensory VPM/VPL and the motor ventrolateral nucleus. Finally, the absence of giant RL terminals defined the borders of the medial pulvinar.

\section{Discussion}

In the present study, we mapped the type and origin of excitatory driver inputs in the macaque thalamus. In contrast to the general assumption that the major role of the thalamus is to relay peripheral information to the cortex, our data show that the larger part of the primate thalamus is devoid of giant vGLUT2-positive terminals. In addition, contrary to the stereotyped view of TC relay cells being driven by the activity of giant excitatory terminals, our data indicate variable composition of the major excitatory/inhibitory afferents. Because the message relayed in the thalamus depends on the size and origin of the driver terminals, the data imply substantial variability in the type of computation performed by different TC cells.
vGLUT1-positive terminals were found only in those nuclei in which cortical axons with large boutons have been described (mediodorsal nucleus and pulvinar). The vGLUT1 immunoreactivity of cortical large terminals was directly demonstrated (Fig. 1 ). These boutons as well as the small cortical layer 6 terminals did not display vGLUT2 immunopositivity, indicating the lack of vGLUT2 in corticothalamic inputs. Third, vGLUT1 and vGLUT2 were not colocalized (Figs. 1, 4, 8), even in regions in which large vGLUT1 and vGLUT2 terminals intermingle, demonstrating distinct origin of the two terminal types. Finally, recent in situ hybridization studies in primates (galago and owl monkey) detected abundant vGLUT1 in the cortex but "absent or very low level of vGLUT2 in layer V and VI" (Balaram et al., 2011; Hackett et al., 2011). Likewise, thalamicprojecting subcortical centers (e.g., inferior and superior colliculus) contained abundant vGLUT2 but negligible vGLUT1 signal (Balaram et al., 2011; Hackett et al., 2011). In the macaque, detection of the mRNA of vGLUTs yielded comparable results (Graziano and Jones, 2008; Alessandro Graziano, personal communication). These data strongly suggest that our conclusions about the distribution, origin, and heterogeneity of the thalamic terminals are not affected by a putative cortical vGLUT2 or subcortical vGLUT1 expression.

\section{Genuine subcortical drive}

Based on their subcortical driver inputs, primary sensory nuclei, the cerebello-recipient thalamus, and the anterior nuclear group were considered as first-order nuclei (Sherman and Guillery, 2006). Our data confirmed these predictions, showing that these nuclei are innervated exclusively and homogeneously by subcortical drivers and receive large excitatory inputs from no other sources. This pattern of innervation forms the morphological basis of the classical thalamic function, the faithful relay of subcortical information to the neocortex.

In addition to first-order nuclei, we also found pure subcortical drive in distinct thalamic zones previously regarded as "higher order" (Sherman and Guillery, 2006), i.e., driven by cortical large terminals. These included the rostral intralaminar group and the medial part of the mediodorsal nucleus with the 


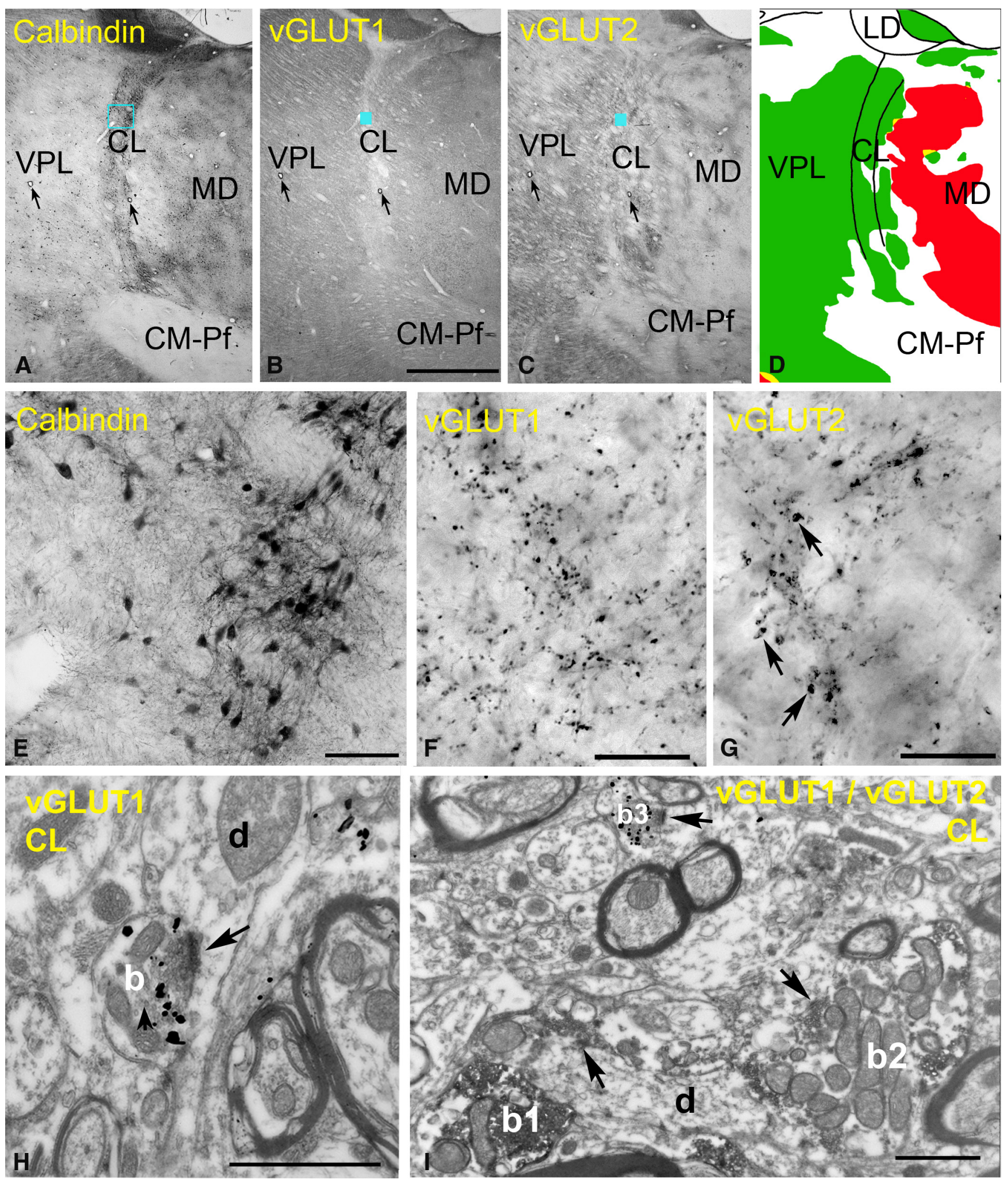

Figure 8. Excitatory inputs of the rostral intralaminar nuclei. $\boldsymbol{A}-\boldsymbol{D}$, Delineation of the intralaminar nuclei. Low-power light micrographs of adjacent sections immunostained for calbindin (A), VGLUT1 (B), and VGLUT2 (C). Note that the borders of the intralaminar nuclei are discernible only in the calbindin immunostaining. Arrows indicate corresponding capillaries. The color map (D) indicate the distribution of large terminals (green, large vGLUT2 terminals; red, large vGLUT1 terminals). Intralaminar nuclei are continuous laterally with VPL but contain no large vGLUT1 terminals, unlike the mediodorsal nucleus. $\boldsymbol{E}-\boldsymbol{G}$, High-power light microscopic images of the blue boxed regions in $\boldsymbol{A}-\boldsymbol{C}$, respectively. Note the lack of large vGLUT1-positive terminals and medium-sized vGLUT2 terminals (arrows in $\boldsymbol{G}$ ) in the centrolateral nucleus. $\boldsymbol{H}$, At the electron microscopic level, a vGLUT1-positive terminal (b) displays RS features. $\boldsymbol{I}$, After double immunostaining, the size difference between RL-type vGLUT2 terminals (b1, b2, DAB precipitate) and RS-type vGLUT1-immunoreactive terminals (b3, silver-intensified gold) are apparent. Scale bars: $A$-C, $2 \mathrm{~mm} ; \boldsymbol{E}, 100 \mu \mathrm{m}$; $\boldsymbol{F}, \mathbf{G}, 20 \mu \mathrm{m} ; \boldsymbol{H}, \mathbf{I}, 1 \mu \mathrm{m}$. 


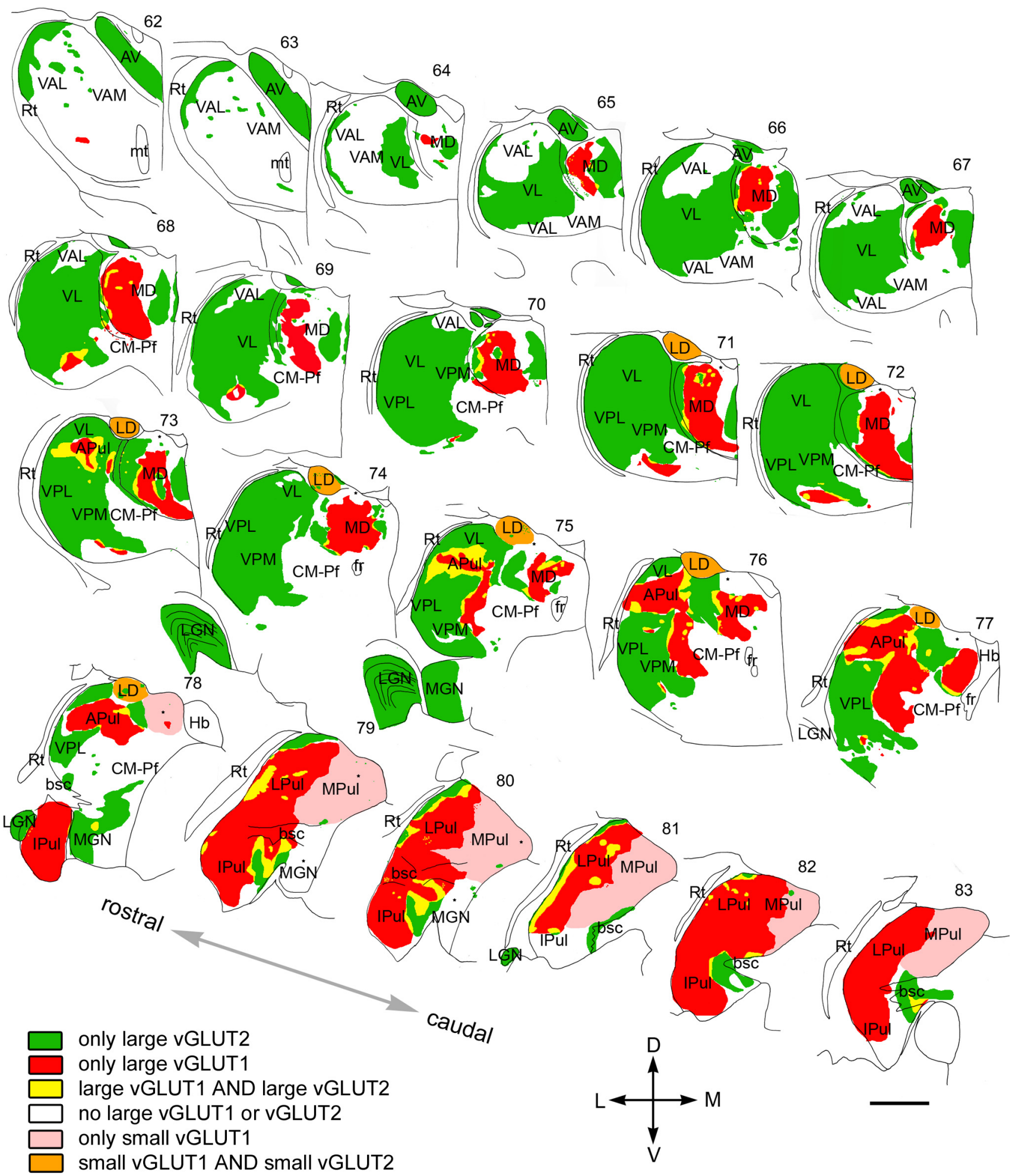

Figure 9. Mosaic of drivers in the primate thalamus. Distribution of cortical and subcortical excitatory terminals in the entire primate thalamus. The map is displayed at 22 coronal levels from anterior (top right) to posterior (bottom left) with $500 \mu \mathrm{m}$ intervals. It is based on observations from seven monkeys. Six different types of innervation pattern are distinguished based on the light and electron microscopic data. Large thalamic territories receive exclusively subcortical (vGLUT2 green) or cortical (vGLUT1, red) RL inputs. Convergence of the two terminal types (yellow) is restricted to border zones. Basal ganglia-recipient nuclei (VA) receive no discernible excitatory RL driver input (white). Cortical input from smaller RL drivers (pink) characterizes medial pulvinar and CM-Pf. Finally, in the laterodorsal nucleus, the convergence of small cortical and small subcortical RL terminals were observed (orange). Single nuclei, especially the pulvinar and the mediodorsal thalamic nuclei, displayed considerable subnuclear heterogeneity in their driver distribution. Small dots indicate clusters of small vGLUT2-positive nerve endings encountered at the light microscopic level. APul, Anterior pulvinar; AV, anteroventral; bsc, brachium of superior colliculus; CM-Pf, centromedian-parafascicular; fr, fasciculus retroflexus; Hb, habenula; Ipul, inferior pulvinar; LD, laterodorsal; LGN, lateral geniculate nucleus; LPul, lateral pulvinar; MD, mediodorsal; MGN, medial geniculate nucleus; MPul, medial pulvinar; mt, mammillothalamic tract; VA, ventral anterior; VL, ventrolateral; VPL, ventral posterolateral; VPM, ventral posteromedial; Rt, reticular thalamus. Scale bar, $2 \mathrm{~mm}$. 
adjacent midline nuclei. Both nuclear groups have been shown to receive subcortical nociceptive afferents (Krout et al., 2002). Thus, the present data underlie the importance of subcortical drive in these nuclei, which may participate in the transmission of abnormal peripheral signals to anterior cingulate cortex during chronic pain (Price, 2000; Krout et al., 2002).

\section{Genuine cortical drive}

Our data unambiguously demonstrated that significant thalamic territories are completely free of subcortical drivers and their RL type terminals have exclusively cortical origin in the primate thalamus (Figs. 3, 9). This indicates that relay cell activity in these regions is only driven by the output of cortical layer 5 pyramidal cells and not by subcortical centers. Recent data showed that corticothalamic RL terminals are as efficient as subcortical drivers and that the physiological properties of cortical and subcortical drivers are identical (Reichova and Sherman, 2004; Groh et al., 2008). Because thalamic neurons driven by cortical layer 5 project back to cortex, this top-down drive can form the basis of a corticocortical information transfer via the thalamus, as shown previously (Theyel et al., 2010), and can mediate higher-order sensory procession, which requires coordinated control of topdown and bottom-up signal flows (Purushothaman et al., 2012). The exact function of this pathway is hotly debated, but our data point out that a significant portion of the primate thalamus is dedicated entirely to this function.

Pure cortical drive was found in the central part of the mediodorsal nucleus and the pulvinar. The pulvinar has been shown to project to a wide range of frontoparietal cortices, indicating its role in linking distinct cortical areas (Cappe et al., 2009). Indeed, small lesions of the pulvinar do not affect perception of elementary information but do interfere with the binding of such information into a single object (Ward et al., 2002). A similar coordinative role has been assigned to the mediodorsal nucleus regarding frontal functions. In cases of schizophrenia, the mediodorsal nucleus and pulvinar display significant cell and volume loss (Cronenwett and Csernansky, 2010; Janssen et al., 2012), which may have a causal relationship with the disorganized frontal functions of these patients as consequence of damage to the cortico-thalamo-cortical pathway.

\section{Convergent zones}

At the boundaries of first- and higher-order territories, large excitatory terminals of cortical and subcortical origin were observed in close proximity (Fig. 4). Because the thalamus is organized in a topographic manner, relay cells innervated by these terminals most probably project to the same cortical territory. This indicates that cortical neurons postsynaptic to these thalamic cells are in a unique position to receive the convergence of information originating from the classical thalamic route (periphery-thalamus-cortex), as well as the trans-thalamic route (cortex-thalamus-cortex). The role of this convergence has still to be determined, but cortical neurons receiving inputs from relay cells activated by bottom-up and top-down drivers may serve to detect coincident sensory and motor activity.

\section{Lack of large excitatory drivers}

In the thalamic territories contacted by the output of the basal ganglia (ventral anterior nucleus), no multisynaptic excitatory driver input (RL-type) could be detected. The proximal dendritic domains, traditionally contacted by excitatory drivers, were innervated by large inhibitory terminals originating from the basal ganglia (Kultas-Ilinsky and Ilinsky, 1990; Kultas-Ilinsky et al.,
1997; Bodor et al., 2008). All basal ganglia terminals form multisynaptic contacts with the proximal dendrites of relay cells (Bodor et al., 2008) and are highly efficient. They are able to maintain inhibitory charge transfer, without depression, at high presynaptic firing rate (Wanaverbecq et al., 2008). Activation of single fiber is able to induce rebound bursts in the postsynaptic thalamic neuron (Bokor et al., 2005). This indicates that the GABAergic pathway, which carries the final computation of the basal ganglia circuit, may have a profound effect on thalamic relay cell activity. The lack of multisynaptic excitatory terminals coupled with the presence of multisynaptic GABAergic terminals in the proximal dendrites of relay cells strongly suggests that the principles of thalamic relay in these nuclei differ from those neurons receiving only multisynaptic excitatory terminals. The strong GABAergic influence on these parts of the thalamus may explain the faithful relay of aberrant basal ganglia activity to the neocortex via the thalamus in Parkinson's disease.

\section{Variability of terminal size}

Thalamic nuclei displayed large heterogeneity in the size and complexity of their driver terminals (Figs. 7, 9). These terminals can be arranged along a continuum from the truly giant cortical and subcortical drivers to the very small cortical terminals establishing a single synapse. Between these two extremes, smaller but still multisynaptic terminals were abundant in several nuclei, in the case of both cortical and subcortical drivers. Small RL-type terminals of cortical origin have been described in the medial pulvinar (Darian-Smith et al., 1999) and the CM-Pf (Balercia et al., 1996) of macaque, which confirms our data. Proper morphological characterization of these terminals requires 3D electron microscopic reconstruction of a representative population of boutons from several thalamic nuclei, which is beyond the scope of the present study. Our data, however, are sufficiently detailed to establish that the size and complexity of drivers are highly variable in the thalamus and indicate that careful consideration of driver size diversity is relevant for an understanding of information transfer in TC circuits. The fact that drivers are smaller in several thalamic territories does not mean that these territories receive only one type of excitatory inputs. Rather, our data together with previous tract tracing studies (Rockland, 1996; Kultas-Ilinsky et al., 2003) demonstrate that small layer 6 cortical terminals are always present together with the drivers of various sizes.

What may be the functional relevance of the driver heterogeneity? The two most important characteristics of traditional TC information relay are the high probability of spike transfer for single stimuli and the rapid depression of this response for repetitive activation (Deschênes et al., 2003; Groh et al., 2008; Budisantoso et al., 2012). This latter feature introduces an important temporal filtering of driver signals at the thalamic level. Both features can be directly linked to the 3D morphology of the large terminals. The high probability of release and the high number of release sites converging on a single dendrite (Mason et al., 1996; Budisantoso et al., 2012) ensures a faithful and large initial response. Conversely, the complex glomerular structure restricts transmitter diffusion and induces AMPA receptor desensitization in the neighboring, closely spaced release sites, which is responsible for the diminished subsequent responses (Budisantoso et al., 2012). This means that the exact size and shape of the thalamic terminals significantly affect the properties of synaptic transmission, as has been shown for complex cerebellar terminals as well (Cathala et al., 2005). Based on these data and on the continuum of RL-type terminals from the very simple to the 
highly complex type we found in the macaque thalamus, we suggest that the properties of TC information transmission will be variable and influenced by the exact size and complexity of RLtype terminal.

Small RL terminals of cortical origin were abundant, e.g., in the medial pulvinar. Recently, Rosenberg et al. (2006) demonstrated that, during temporal lobe seizures that propagate from one cortical region to another, the medial pulvinar is always involved. This indicates that pure cortical drive via small RL terminals can play an important role in the propagation of pathological activity.

\section{Conclusions}

A recent review indicates a substantial diversity in TC axonal output pattern (Clascá et al., 2012). Our data here disclosed large heterogeneity in the organization of the major inputs to the thalamus. This indicates that the principles of operation in the thalamus do not follow a simple logic but express significant regional heterogeneity both in the input and output sides.

\section{References}

Apkarian AV, Shi T (1994) Squirrel monkey lateral thalamus. I. Somatic nociresponsive neurons and their relation to spinothalamic terminals. J Neurosci 14:6779-6795. Medline

Balaram P, Hackett TA, Kaas JH (2011) VGLUT1 mRNA and protein expression in the visual system of prosimian galagos (Otolemur garnetti). Eye Brain 2011:81-98. CrossRef Medline

Balercia G, Kultas-Ilinsky K, Bentivoglio M, Ilinsky IA (1996) Neuronal and synaptic organization of the centromedian nucleus of the monkey thalamus: a quantitative ultrastructural study, with tract tracing and immunohistochemical observations. J Neurocytol 25:267-288. CrossRef Medline

Barthó P, Freund TF, Acsády L (2002) Selective GABAergic innervation of thalamic nuclei from zona incerta. Eur J Neurosci 16:999-1014. CrossRef Medline

Beggs J, Jordan S, Ericson AC, Blomqvist A, Craig AD (2003) Synaptology of trigemino- and spinothalamic lamina I terminations in the posterior ventral medial nucleus of the macaque. J Comp Neurol 459:334-354. CrossRef Medline

Berod A, Hartman BK, Pujol JF (1981) Importance of fixation in immunohistochemistry: use of formaldehyde solutions at variable $\mathrm{pH}$ for the localization of tyrosine hydroxylase. J Histochem Cytochem 29:844-850. CrossRef Medline

Bodor AL, Giber K, Rovó Z, Ulbert I, Acsády L (2008) Structural correlates of efficient GABAergic transmission in the basal ganglia-thalamus pathway. J Neurosci 28:3090-3102. CrossRef Medline

Boivie J (1979) An anatomical reinvestigation of the termination of the spinothalamic tract in the monkey. J Comp Neurol 186:343-369. CrossRef Medline

Bokor H, Frère SGA, Eyre MD, Slézia A, Ulbert I, Lüthi A, Acsády L (2005) Selective GABAergic control of higher-order thalamic relays. Neuron 45: 929-940. CrossRef Medline

Bourassa J, Deschênes M (1995) Corticothalamic projections from the primary visual cortex in rats: a single fiber study using biocytin as an anterograde tracer. Neuroscience 66:253-263. CrossRef Medline

Bourassa J, Pinault D, Deschênes M (1995) Corticothalamic projections from the cortical barrel field to the somatosensory thalamus in rats: a single-fibre study using biocytin as an anterograde tracer. Eur J Neurosci 7:19-30. CrossRef Medline

Budisantoso T, Matsui K, Kamasawa N, Fukazawa Y, Shigemoto R (2012) Mechanisms underlying signal filtering at a multisynapse contact. J Neurosci 32:2357-2376. CrossRef Medline

Cappe C, Morel A, Rouiller EM (2007) Thalamocortical corticothalamic parietal cortex and the dual pattern of projections of the posterior in macaque monkeys. Neuroscience 146:1371-1387. CrossRef Medline

Cappe C, Morel A, Barone P, Rouiller EM (2009) The thalamocortical projection systems in primate: an anatomical support for multisensory and sensorimotor interplay. Cereb Cortex 19:2025-2037. CrossRef Medline

Castaigne P, Lhermitte F, Buge A, Escourolle R, Hauw JJ, Lyon-Caen O (1981) Paramedian thalamic and midbrain infarct: clinical and neuropathological study. Ann Neurol 10:127-148. CrossRef Medline
Cathala L, Holderith NB, Nusser Z, DiGregorio DA, Cull-Candy SG (2005) Changes in synaptic structure underlie the developmental speeding of AMPA receptor-mediated EPSCs. Nat Neurosci 8:1310-1318. CrossRef Medline

Clascá F, Rubio-Garrido P, Jabaudon D (2012) Unveiling the diversity of thalamocortical neuron subtypes. Eur J Neurosci 35:1524-1532. CrossRef Medline

Colonnier M, Guillery RW (1964) Synaptic organization in the lateral geniculate nucleus of the monkey. Z Zellforsch Mikrosk Anat 62:333-355. CrossRef Medline

Cronenwett WJ, Csernansky J (2010) Thalamic pathology in schizophrenia. Curr Top Behav Neurosci 4:509-528. CrossRef Medline

Darian-Smith C, Tan A, Edwards S (1999) Comparing thalamocortical and corticothalamic microstructure and spatial reciprocity in the macaque ventral posterolateral nucleus (VPLc) and medial pulvinar. J Comp Neurol 410:211-234. CrossRef Medline

Deschênes M, Bourassa J, Pinault D (1994) Corticothalamic projections from layer $\mathrm{V}$ cells in rat are collaterals of long-range corticofugal axons. Brain Res 664:215-219. CrossRef Medline

Deschênes M, Timofeeva E, Lavallée P (2003) The relay of high-frequency sensory signals in the whisker-to-barreloid pathway. J Neurosci 23:6778 6787. Medline

Fisher R, Salanova V, Witt T, Worth R, Henry T, Gross R, Oommen K, Osorio I, Nazzaro J, Labar D, Kaplitt M, Sperling M, Sandok E, Neal J, Handforth A, Stern J, DeSalles A, Chung S, Shetter A, Bergen D, et al. (2010) Electrical stimulation of the anterior nucleus of thalamus for treatment of refractory epilepsy. Epilepsia 51:899-908. CrossRef Medline

Fremeau RT Jr, Troyer MD, Pahner I, Nygaard GO, Tran CH, Reimer RJ, Bellocchio EE, Fortin D, Storm-Mathisen J, Edwards RH (2001) The expression of vesicular glutamate transporters defines two classes of excitatory synapse. Neuron 31:247-260. CrossRef Medline

Graziano A, Jones EG (2008) Immunocytochemical dissection of the corematrix thalamocortical system in monkeys. Soc Neurosci Abstr 34:370.7.

Graziano A, Liu XB, Murray KD, Jones EG (2008) Vesicular glutamate transporters define two sets of glutamatergic afferents to the somatosensory thalamus and two thalamocortical projections in the mouse. J Comp Neurol 507:1258-1276. CrossRef Medline

Groh A, de Kock CP, Wimmer VC, Sakmann B, Kuner T (2008) Driver or coincidence detector: modal switch of a corticothalamic giant synapse controlled by spontaneous activity and short-term depression. J Neurosci 28:9652-9663. CrossRef

Hackett TA, Takahata T, Balaram P (2011) VGLUT1 and VGLUT2 mRNA expression in the primate auditory pathway. Hear Res 274:129-141. CrossRef Medline

Hamani C, Schwalb JM, Rezai AR, Dostrovsky JO, Davis KD, Lozano AM (2006) Deep brain stimulation for chronic neuropathic pain: long-term outcome and the incidence of insertional effect. Pain 125:188-196. CrossRef Medline

Harting JK, Huerta MF, Frankfurter AJ, Strominger NL, Royce GJ (1980) Ascending pathways from the monkey superior colliculus: an autoradiographic analysis. J Comp Neurol 192:853-882. CrossRef Medline

Herzog E, Bellenchi GC, Gras C, Bernard V, Ravassard P, Bedet C, Gasnier B, Giros B, El Mestikawy S (2001) The existence of a second vesicular glutamate transporter specifies subpopulations of glutamatergic neurons. J Neurosci 21:RC181(1-6). Medline

Ilinsky IA, Jouandet ML, Goldman-Rakic PS (1985) Organization of the nigrothalamocortical system in the rhesus monkey. J Comp Neurol 236: 315-330. CrossRef Medline

Janssen J, Alemán-Gómez Y, Reig S, Schnack HG, Parellada M, Graell M, Moreno C, Moreno D, Mateos-Pérez JM, Udias JM, Arango C, Desco M (2012) Regional specificity of thalamic volume deficits in male adolescents with early-onset psychosis. Br J Psychiatry 200:30-36. CrossRef Medline

Jones E (2007) Individual thalamic nuclei. In: The thalamus, Pt IV. Cambridge, UK: Cambridge UP.

Jones EG, Powell TP (1969) An electron microscopic study of the mode of termination of cortico-thalamic fibres within the sensory relay nuclei of the thalamus. Proc R Soc Lond B Biol Sci 172:173-185. CrossRef Medline

Krout KE, Belzer RE, Loewy AD (2002) Brainstem projections to midline and intralaminar thalamic nuclei of the rat. J Comp Neurol 448:53-101. CrossRef Medline

Kultas-Ilinsky K, Ilinsky IA (1990) Fine structure of the magnocellular sub- 
division of the ventral anterior thalamic nucleus (VAmc) of Macaca mulatta. II. Organization of nigrothalamic afferents as revealed with EM autoradiography. J Comp Neurol 294:479-489. CrossRef Medline

Kultas-Ilinsky K, Reising L, Yi H, Ilinsky IA (1997) Pallidal afferent territory of the Macaca mulatta thalamus: neuronal and synaptic organization of the VAdc. J Comp Neurol 386:573-600. CrossRef Medline

Kultas-Ilinsky K, Sivan-Loukianova E, Ilinsky IA (2003) Reevaluation of the primary motor cortex connections with the thalamus in primates. J Comp Neurol 457:133-158. CrossRef Medline

Land PW, Kyonka E, Shamalla-Hannah L (2004) Vesicular glutamate transporters in the lateral geniculate nucleus: expression of VGLUT2 by retinal terminals. Brain Res 996:251-254. CrossRef Medline

Laureys S, Faymonville ME, Luxen A, Lamy M, Franck G, Maquet P (2000) Restoration of thalamocortical connectivity after recovery from persistent vegetative state. Lancet 355:1790-1791. CrossRef Medline

Lavallée P, Urbain N, Dufresne C, Bokor H, Acsády L, Deschênes M (2005) Feedforward inhibitory control of sensory information in higher-order thalamic nuclei. J Neurosci 25:7489-7498. CrossRef Medline

Limousin P, Speelman JD, Gielen F, Janssens M (1999) Multicentre European study of thalamic stimulation in parkinsonian and essential tremor. J Neurol Neurosurg Psychiatry 66:289-296. CrossRef Medline

Mason A, Ilinsky IA, Beck S, Kultas-Ilinsky K (1996) Reevaluation of synaptic relationships of cerebellar terminals in the ventral lateral nucleus of the rhesus monkey thalamus based on serial section analysis and threedimensional reconstruction. Exp Brain Res 109:219-239. CrossRef Medline

Masterson SP, Li J, Bickford ME (2009) Synaptic organization of the tectorecipient zone of the rat lateral posterior nucleus. J Comp Neurol 515: 647-663. CrossRef Medline

Molinari M, Dell'Anna ME, Rausell E, Leggio MG, Hashikawa T, Jones EG (1995) Auditory thalamocortical pathways defined in monkeys by calcium-binding protein immunoreactivity. J Comp Neurol 362: 171-194. CrossRef Medline

Paxinos G, Huang X, Toga A (2000) The rhesus monkey brain in stereotaxic coordinates. San Diego: Academic.

Percheron G, François C, Talbi B, Yelnik J, Fénelon G (1996) The primate motor thalamus. Brain Res Rev 22:93-181. CrossRef Medline

Price DD (2000) Psychological and neural mechanisms of the affective dimension of pain. Science 288:1769-1772. CrossRef Medline

Purushothaman G, Marion R, Li K, Casagrande VA (2012) Gating and control of primary visual cortex by pulvinar. Nat Neurosci 15:905-912. Medline

Ralston HJ 3rd, Herman MM (1969) The fine structure of neurons and synapses in ventrobasal thalamus of the cat. Brain Res 14:77-97. CrossRef Medline

Rausell E, Bae CS, Viñuela A, Huntley GW, Jones EG (1992) Calbindin and parvalbumin cells in monkey VPL thalamic nucleus: distribution, laminar cortical projections, and relations to spinothalamic terminations. J Neurosci 12:4088-4111. Medline

Reichova I, Sherman SM (2004) Somatosensory corticothalamic projections: distinguishing drivers from modulators. J Neurophysiol 92:21852197. CrossRef Medline

Rockland KS (1996) Two types of corticopulvinar terminations: round (type 2) and elongate (type 1). J Comp Neurol 368:57-87. CrossRef Medline

Rosenberg DS, Mauguière F, Demarquay G, Ryvlin P, Isnard J, Fischer C,
Guénot M, Magnin M (2006) Involvement of medial pulvinar thalamic nucleus in human temporal lobe seizures. Epilepsia 47:98-107.

Rouiller EM, Welker E (2000) A comparative analysis of the morphology of corticothalamic projections in mammals. Brain Res Bull 53:727-741. CrossRef Medline

Rouiller EM, Liang F, Babalian A, Moret V, Wiesendanger M (1994) Cerebellothalamocortical and pallidothalamocortical projections to the primary and supplementary motor cortical areas: a multiple tracing study in macaque monkeys. J Comp Neurol 345:185-213. CrossRef Medline

Schiff ND, Giacino JT, Kalmar K, Victor JD, Baker K, Gerber M, Fritz B, Eisenberg B, Biondi T, O'Connor J, Kobylarz EJ, Farris S, Machado A, McCagg C, Plum F, Fins JJ, Rezai AR (2007) Behavioural improvements with thalamic stimulation after severe traumatic brain injury. Nature 448 : 600-603. CrossRef Medline

Schnell SA, Staines WA, Wessendorf MW (1999) Reduction of lipofuscinlike autofluorescence in fluorescently labeled tissue. J Histochem Cytochem 47:719-730. CrossRef Medline

Schwartz ML, Dekker JJ, Goldman-Rakic PS (1991) Dual mode of corticothalamic synaptic termination in the mediodorsal nucleus of the rhesus monkey. J Comp Neurol 309:289-304. CrossRef Medline

Sherman SM, Guillery RW (1996) Functional organization of thalamocortical relays. J Neurophysiol 76:1367-1395. Medline

Sherman SM, Guillery RW (1998) On the actions that one nerve cell can have on another: distinguishing "drivers" from "modulators." Proc Natl Acad Sci U S A 95:7121-7126. CrossRef

Sherman SM, Guillery RW (2006) Exploring the thalamus and its role in cortical function. Cambridge, MA: Massachusetts Institute of Technology.

Somogyi P, Hodgson AJ (1985) Antisera to gamma-aminobutyric acid. III. Demonstration of GABA in Golgi-impregnated neurons and in conventional electron microscopic sections of cat striate cortex. J Histochem Cytochem 33:249-257. CrossRef Medline

Taktakishvili O, Sivan-Loukianova E, Kultas-Ilinsky K, Ilinsky IA (2002) Posterior parietal cortex projections to the ventral lateral and some association thalamic nuclei in Macaca mulatta. Brain Res Bull 59:135-150. CrossRef Medline

Theyel BB, Llano DA, Sherman SM (2010) The corticothalamocortical circuit drives higher-order cortex in the mouse. Nat Neurosci 13:84-88. CrossRef Medline

Veinante P, Lavallée P, Deschênes M (2000) Corticothalamic projections from layer 5 of the vibrissal barrel cortex in the rat. J Comp Neurol 424:197-204. CrossRef Medline

Vicq d'Azyr F (1786) Traite d'anatomie et de physiologie. Paris: Didot.

Wanaverbecq N, Bodor AL, Bokor H, Slézia A, Lüthi A, Acsády L (2008) Contrasting the functional properties of GABAergic axon terminals with single and multiple synapses in the thalamus. J Neurosci 28:11848-11861. CrossRef Medline

Ward R, Danziger S, Owen V, Rafal R (2002) Deficits in spatial coding and feature binding following damage to spatiotopic maps in the human pulvinar. Nat Neurosci 5:99-100. CrossRef Medline

Wilson JR (1989) Synaptic organization of individual neurons in the macaque lateral geniculate nucleus. J Neurosci 9:2931-2953. Medline

Yamamoto T, Katayama Y, Kobayashi K, Oshima H, Fukaya C, Tsubokawa T (2010) Deep brain stimulation for the treatment of vegetative state. Eur J Neurosci 32:1145-1151. CrossRef Medline 